\title{
S-bearing molecules in massive dense cores ${ }^{\star} \star \star$
}

\author{
F. Herpin ${ }^{1,2}$, M. Marseille ${ }^{3}$, V. Wakelam ${ }^{1,2}$, S. Bontemps ${ }^{1,2}$, and D. C. Lis ${ }^{4}$ \\ 1 Université de Bordeaux, Laboratoire d'Astrophysique de Bordeaux, 33000 Bordeaux, France \\ e-mail: herpin@obs.u-bordeaux1.fr \\ 2 CNRS/INSU, UMR 5804, BP 89, 33271 Floirac Cedex, France \\ SRON Netherlands Institute for Space Research, Landleven 12, 9747AD Groningen, The Netherlands \\ ${ }^{4}$ California Institute of Technology, Downs Laboratory of Physics 320-47, Pasadena, CA 91125, USA
}

Received 30 October 2008 / Accepted 28 May 2009

\begin{abstract}
Context. Although few in number, high-mass stars play a major role in the interstellar energy budget and the shaping of the Galactic environment; however, the formation of high-mass stars is not well understood, because of their large distances, short time scales, and heavy extinction.

Aims. The chemical composition of the massive cores forming high-mass stars can put some constraints on the time scale of the massive star formation: sulfur chemistry is of specific interest thanks to its rapid evolution in warm gas and because the abundance of sulfur-bearing species increases significantly with the temperature.

Methods. Two mid-infrared quiet and two brighter massive cores were observed in various transitions ( $E_{\text {up }}$ up to $289 \mathrm{~K}$ ) of CS, OCS, $\mathrm{H}_{2} \mathrm{~S}, \mathrm{SO}$, and $\mathrm{SO}_{2}$ and of their ${ }^{34} \mathrm{~S}$ isotopologues at $\mathrm{mm}$ wavelengths with the IRAM $30 \mathrm{~m}$ and CSO telescopes. The 1D modeling of the dust continuum is used to derive the density and temperature laws, which were then applied in the RATRAN code to modeling the observed line emission and to deriving the relative abundances of the molecules.

Results. All lines are detected, except the highest energy $\mathrm{SO}_{2}$ transition. Infall (up to $2.9 \mathrm{~km} \mathrm{~s}^{-1}$ ) may be detected towards the core W43MM1. The inferred mass rate is 5.8-9.4 $10^{-2} M_{\odot} /$ yr. We propose an evolutionary sequence of our sources $(\mathrm{W} 43 \mathrm{MM} 1 \rightarrow$ IRAS18264-1152 $\rightarrow$ IRAS05358+3543 $\rightarrow$ IRAS18162-2048), based on the SED analysis. The analysis of the variations in abundance ratios from source to source reveals that the $\mathrm{SO}$ and $\mathrm{SO}_{2}$ relative abundances increase with time, while $\mathrm{CS}$ and OCS decrease.

Conclusions. Molecular ratios, such as $\left[\mathrm{OCS} / \mathrm{H}_{2} \mathrm{~S}\right],\left[\mathrm{CS} / \mathrm{H}_{2} \mathrm{~S}\right]$, [SO/OCS], $\left[\mathrm{SO}_{2} / \mathrm{OCS}\right],[\mathrm{CS} / \mathrm{SO}]$, and $\left[\mathrm{SO}_{2} / \mathrm{SO}\right.$ may be good indicators of evolution, depending on layers probed by the observed molecular transitions. Observations of molecular emission from warmer layers, so that involving higher upper energy levels must be included.
\end{abstract}

Key words. ISM: individual objects: W43MM1, IRAS18264-1152, IRAS05358+3543, IRAS18162-2048 - ISM: abundances stars: formation - line: profiles

\section{Introduction}

The OB stars are the main contributors to the evolution and energy budget of galaxies. Their formation, however, has not been understood yet and the classical scheme for low-mass star formation (see Andre et al. 2000, and references therein) cannot be applied as such to OB stars. Indeed, young OB stars and protostars strongly interact with the surrounding massive clouds and cores, leading to a complex and still not clearly defined sequence of objects from pre-stellar cores that are often believed to be hosted in the so-called IR dark clouds (IRDCs), to high-mass protostellar objects (HMPOs), to hot cores and uItra compact HII regions (e.g. Beuther et al. 2007a; Menten et al. 2005).

This tentative evolutionary scheme is, however, mostly based on observations of distant objects, for which the physical scales are often about $0.5 \mathrm{pc}$. In contrast, the well-established protostellar evolution from Class 0 to Class III young stellar objects (YSOs) for low-mass stars has revealed that the envelopes of

\footnotetext{
* Fits files are only available in electronic form at the CDS via anonymous ftp to cdsarc.u-strasbg.fr (130.79.128.5) or via

http://cdsweb.u-strasbg.fr/cgi-bin/qcat?J/A+A/504/853

$\star \star$ Tables 6-10 and Figs. 14-17 are only available in electronic form at http://www . aanda.org
}

individual protostars have typical sizes ranging from 0.02 to $0.04 \mathrm{pc}$ in clustered star-forming regions (see Motte \& André 2001, and references therein). HMPOs are therefore more protoclusters than individual protostars. Recent interferometric observations have confirmed that they are fragmented and that they actually form clusters of stars (e.g. Beuther \& Schilke 2004; Shepherd et al. 2003). Motte et al. (2007) have obtained a complete view of high-mass protostellar phases on the scale of a giant molecular cloud that is not too distant (Cygnus X at $1.7 \mathrm{kpc}$ ). This complex is rich enough to provide a first reliable statistic (40 massive dense cores) on a physical scale of $\sim 0.1 \mathrm{pc}$.

From this unbiased survey of the earliest phases of the highmass star formation, it has been deduced that massive pre-stellar dense cores are extremely rare, and that of the order of $50 \%$ of the massive dense cores are already forming high-mass stars while being still cold and not bright in the IR (IR-quiet dense cores). The remaining 50\% of dense massive cores can be called for IR-bright dense cores. Referring to the typical sizes discussed in Williams et al. (2000), these 0.1 pc size objects are dense cores, in contrast to clumps that are more $1 \mathrm{pc}$ size. On the other hand, these $0.1 \mathrm{pc}$ dense cores are not necessarily precursors of single stars and may form a group of stars, so would be considered as clumps in the primary definition of 
Table 1. Source sample.

\begin{tabular}{|c|c|c|c|c|c|c|}
\hline \multirow[b]{2}{*}{ Source } & \multicolumn{3}{|c|}{ Source Coordinates } & \multicolumn{2}{|c|}{ Properties } & \multirow{2}{*}{$\begin{array}{c}F_{21}{ }^{a} \\
(\mathrm{Jy})\end{array}$} \\
\hline & $\begin{array}{l}\text { RA (J2000.0) } \\
(\mathrm{h} \mathrm{m} \mathrm{s})\end{array}$ & $\begin{array}{l}\text { Dec } \\
\left({ }^{\circ}, \prime \prime\right)\end{array}$ & $\begin{array}{l}V_{\mathrm{LSR}} \\
\left(\mathrm{km} \mathrm{s}^{-1}\right)\end{array}$ & $\begin{array}{l}L_{\mathrm{bol}} \\
\left(L_{\odot}\right)\end{array}$ & $\begin{array}{c}d \\
(\mathrm{kpc})\end{array}$ & \\
\hline IRAS05358+3543 & & & & $6.3 \times 10^{3}$ & 1. & 18.9 \\
\hline IRAS18162-2048 & 181912.1 & -204731 & +12.1 & $2.0 \times 10^{4}$ & 1.9 & 141.4 \\
\hline IRAS18264-1152 & 182914.4 & -115023 & +43.6 & $1 . \times 10^{4}$ & 3.5 & 4.9 \\
\hline W43MM1 & 184747.0 & -015428 & +98.8 & $2.3 \times 10^{4}$ & 5.5 & 4.8 \\
\hline
\end{tabular}

The $F_{21}$ value is the MSX value for the $21 \mu \mathrm{m}$ flux, corrected for the distance of each source $\left(F_{21} \times(d / 1.7)^{2}\right)$.

Williams et al. (2000). We follow the scheme discussed in Motte \& Hennebelle (2009), in which the study of massive dense cores on physical scales of $\sim 0.1 \mathrm{pc}$ or less is required to really address the question of evolutionary stages for high-mass star and cluster formation. The interest in studying the evolution of massive dense cores is also supported by the most recent theoretical scenarios, which predict either a monolithic collapse of turbulent gas on the scale of massive dense cores (Tan \& McKee 2002; Krumholz \& McKee 2008), or a competitive accretion inside the gravitational potential of a cluster-forming massive dense core (Bonnell \& Bate 2006). Finally, it is worth noting that some direct observational evidence of the so-called global collapse has usually been obtained for massive dense cores on the typical $\sim 0.1$ pc scale (e.g. Motte et al. 2005).

The chemical composition of the protostellar envelopes can be used to constrain the ages of low-mass protostars (Millar et al. 1997; Hatchell et al. 1998; Doty et al. 2002; Wakelam et al. 2004a). The sulfur chemistry is of specific interest because of its rapid evolution in warm gas and of the abundances of sulfurbearing species increasing significantly with temperature, both by ice evaporation and by shock interaction. Sulfur could thus act as a clock on time scales relevant to the embedded phase of star formation (Charnley 1997; Wakelam et al. 2004a,b). A good sketch has now been established for these objects allowing them to be dated within a "class" of objects. Here we propose some first constraints on the time of evolution of the targeted massive cores; detailed chemical modeling will be presented in a forthcoming paper (Wakelam et al., in preparation).

For high-mass protostars, the first studies using sulfurbearing molecules as evolutionary tracers are reported in Hatchell et al. (1998) and van der Tak et al. (2003). We present here a complementary study of sources, which are weaker at mid-infrared wavelengths. Also in contrast to van der Tak et al. (2003) who used chemical models without the inclusion of atomic oxygen (e.g., Charnley 1997), we prefer to use the recent results of Lis et al. (2001) and Vastel et al. (2002). Furthermore, Wakelam et al. (2004b) underlined that the sulfur may be mainly in the atomic form in hot cores.

To analyze these observations, we apply a similar method to Wakelam et al. (2004a), who measured the age of the hot corinos of IRAS16293-2422 with reasonable success. Our observations of low- and high-energy transitions for each selected molecule enable us to probe the inner $(>100 \mathrm{~K})$ and outer $(<100 \mathrm{~K})$ regions of the objects studied. We first use a radiative transfer model (RATRAN, Hogerheijde \& van der Tak 2000) to constrain the sulfur-bearing abundance profiles within the envelopes (this paper). In a forthcoming paper, the derived abundance profiles will be compared to a grid of chemical models (NAHOON) adapted to the physical structure of each source in order to constrain the age of each source. To cover the complete range of energy of the massive envelopes (from $20 \mathrm{~K}$ to more than $100 \mathrm{~K}$, Doty et al. 2002), transitions with upper energies between 10 and $110 \mathrm{~cm}^{-1}$ are required. Indeed, even if observing emission lines covering a broad energy range is mandatory for deriving the excitation conditions of the molecules, the only way to probe the inner warmer regions is to observe high-energy transitions whose levels are not populated in the cold outer gas. Actually, probing the inner region of massive dense cores is the only way to chemically date their evolution from the molecular cloud.

\section{The source sample}

The source sample is given in Table 1: luminosity, distance, and velocities are from Sridharan et al. (2002), Benedettini et al. (2004), and Motte et al. (2003), respectively, for IRAS05358+3543 and IRAS18264-1152, IRAS18162-2048, and W43MM1. The coordinates correspond to the peak of the mm continuum emission from Beuther et al. (2002a) for IRAS05358+3543 and IRAS18264-1152, and Motte et al. (2003) for W43MM1. For IRAS18162-2048, coordinates correspond to the $\mathrm{CO}$ emission peak from Benedettini et al. (2004). The selected sources are two IR-quiet dense cores MSX Flux $_{21 \mu \mathrm{m}}<10 \mathrm{Jy}$ at $1.7 \mathrm{kpc}$, following the definition of Motte et al. 2007) and two slightly brighter ones (IRAS05358+3543 and IRAS18162-2048), with bolometric luminosities $0.6-2.310^{4} L_{\odot}$ at distances $1.8-5.5 \mathrm{kpc}$ (see Table 1) and sizes of $\sim 0.11-0.13 \mathrm{pc}$ (as seen in the beam of our observations, see Sect. 6.1). These massive dense cores are expected to be fragmented on small scales and are meant to represent the earliest phases of the formation of clusters. The expected multiplicity can, however, be neglected to first order, to follow the average chemical evolution of their surrounding dense cores. In fact, a small fraction of the total mass of the cores is in the individual fragments, and usually the large-scale, average-density profile of the cores applies well down to the smallest observed structures. This suggests that the cores could be well represented to first order as spherically symmetric structures from a few $0.1 \mathrm{pc}$ down to $\sim 0.01 \mathrm{pc}$. IRAS $05358+3543$ has for instance been studied with millimeter interferometers (see Leurini et al. 2007). It appears that the $\sim 0.3$ pc size core of $613 M_{\odot}$ splits into two main objects (mmla and mm1b) of 1 and $0.6 M_{\odot}$ inside regions of $0.006 \mathrm{pc}$ diameter, separated only by $\sim 0.008 \mathrm{pc}$. A mass of $1.6 M_{\odot}$ in a region $\sim 0.01 \mathrm{pc}$ comprising mmla and mm1b, inside a $0.3 \mathrm{pc}$ size core of $613 M_{\odot}$ corresponds to an average radial profile of the density of $r^{-1.25}$, which is actually close to the $r^{-1.4}$ profile derived from large scales by Beuther et al. (2002b) and which we use to describe the global 1D distribution of matter in the core. Two other millimeter condensations, $\mathrm{mm} 2$ and $\mathrm{mm} 3$, are also detected with the interferometer. They could eventually confuse the results for molecular tracers that can trace such high-density regions.

A large number of continuum and molecular line $(\mathrm{CO}, \mathrm{SiO}$, $\mathrm{CH}_{3} \mathrm{OH}, \mathrm{H}_{2} \mathrm{CO}$, etc.) data towards these sources have been published (e.g. Benedettini et al. 2004). The physical conditions thus have already been determined in some of the envelopes (e.g. Beuther et al. 2002b), and we use the published data and our own observations. We limited our study to massive dense cores to simplify this first step in the analysis for two reasons. First, different physical conditions were observed in these objects, but they still have the same structure in the sense that there is no strong UV field or ultra-compact HII regions, which will drastically change the chemistry with time. The second reason is that these cores could be the equivalent for massive dense cores of the Class 0 YSOs in the general scenario of gas and dust 
evolution, for which sulfur chemistry (Wakelam et al. 2004a,b) has already been studied. Finally, the sources studied here are part of the sample that will be studied within the key program WISH $^{1}$ with the HIFI instrument aboard the ESA Herschel Space Observatory.

Both IRAS05358+3543 and IRAS18264-1152 are part of the Sridharan et al. (2002) sample. IRAS05358+3543 is a relatively low-luminosity $\left(L \sim 6.3 \times 10^{3} L_{\odot}\right)$ and nearby $(1.8 \mathrm{kpc})$ massive dense core, composed of three main sources ( $\mathrm{mm} 1$, $\mathrm{mm} 2$, and $\mathrm{mm} 3$; total mass around $613 M_{\odot}$ ), all within $4^{\prime \prime} \times 9^{\prime \prime}$ (Leurini et al. 2007), hence within the telescope beam. The source exhibits a hypercompact HII region that may evolve into an UC-HII region. Beuther et al. (2007b) show that the source actually splits into four individual continuum components, two of which are part of a protobinary system, mml (dynamical age $\sim 3.6 \times 10^{4} \mathrm{yr}$ ). According to Longmore et al. (2006), the main mid-IR source coincides with mm1a of Beuther et al. (2007b), but the second mid-IR source does not coincide with the $\mathrm{mm}$ source $\mathrm{mm} 1 \mathrm{~b}$, suggesting even more substructure. The source exhibits a class II methanol maser, indicating that it is a very young object. The source $\mathrm{mm} 3$ is the coldest and youngest ( $T_{\text {dust }} \leq 20 \mathrm{~K}$ ). Several molecular outflows are observed (two from $\mathrm{mm} 1$, outflow rate of $6 \times 10^{4} M_{\odot}$; Beuther et al. 2002a). Strong line-emission asymmetries in $\mathrm{HCO}^{+}$have been observed by Fuller et al. (2005).

IRAS18264-1152 is a massive dense core at a distance of $3.5 \mathrm{kpc}$. Its luminosity is estimated to reach $10^{4} L_{\odot}$. A massive molecular outflow is centered on the mm dust peak (Beuther et al. 2002a). Continuum observations with the Plateau de Bure observatory (Qiu et al. 2007) reveal that the source is double; the mass of the dust-gas core is estimated to be $570 M_{\odot}$, while the outflow mass is $20.2 M_{\odot}$ (dynamical age of $0.5 \times 10^{4} \mathrm{yr}$ ).

Benedettini et al. (2004) performed multiline observations of IRAS18162-2048, a luminous infrared source $\left(L=2 \times 10^{4} L_{\odot}\right.$; Yamashita et al. 1989) at a kinematic distance of $1.9 \mathrm{kpc}$ (Kurtz et al. 1994). This object is associated with the IRAS source and is actually an IR-bright dense core. A very powerful outflow ( $M \sim 570 M_{\odot}$ ), one of the most massive known, was observed (dynamical age $\sim 10^{6} \mathrm{yr}$ ). The source has been resolved into a cluster of several objects (Stecklum et al. 1997).

W43 is a massive star-forming region, located at $5.5 \mathrm{kpc}$, which harbors a giant HII region, and it has been studied in detail by Motte et al. (2003). W43MM1 is the most massive dense core of the region and does not coincide with any known IR source. The dust temperature is estimated to be $19 \mathrm{~K}$ and its luminosity $2.3 \times 10^{4} L_{\odot} . \mathrm{W} 43 \mathrm{MM} 1$ harbors a methanol and a water maser, but does not show any infrared or centimeter emission. An infall has been detected by Motte and collaborators (private communication).

Continuum emission from our sources is determined with well-sampled observations from IRAS, MSX, SCUBA/JCMT, or SHARC/CSO (Hunter et al. 2000; Jenness et al. 1995; Minier et al. 2005), SMA (Beuther et al. 2007b; Su et al. 2004), and MAMBO/IRAM30m (Beuther et al. 2002b). The SED for IRAS18162-2048 is particularly constrained, thanks to a complete ISO-SWS observation. Fewer continuum observations are available for the source W43MM1.

From the fitted SEDs (see Sect. 6.1) shown in Fig. 1, we make a rough evolutionary classification of our 4 objects, using the following parameters:

- wavelength and flux of the maximum continuum emission;

${ }^{1}$ http://www.strw.leidenuniv.nl/WISH/, PI: E. van Dishoeck.

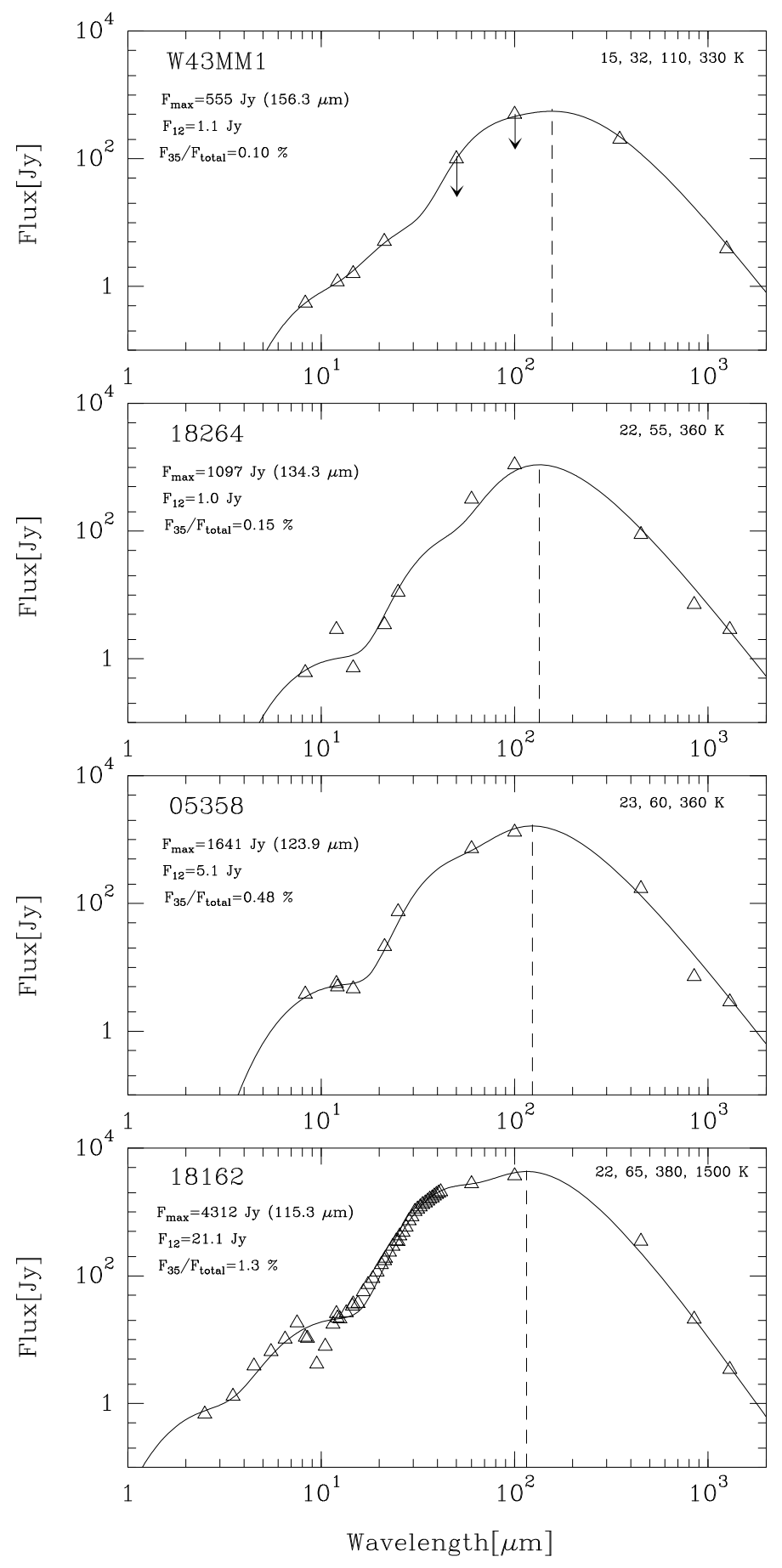

Fig. 1. Spectral energy distributions obtained from 1D model overlaid on observed fluxes of each source. Peak fluxes in the millimeter range have been adjusted to fit radial extension of the model. Each caption gives the temperatures of the black body components (top right), the maximum flux $F_{\max }$, and the corresponding wavelength of the SED, the flux $F_{12}$ at $12 \mu \mathrm{m}$, and the contribution of the hot part $\left(F_{35}\right.$, integrated flux for $\lambda<35 \mu \mathrm{m}$ ) to the total integrated flux $F_{\text {total }}$ (top left).

\section{- flux at $12 \mu \mathrm{m}$; \\ - contribution of the hot part $(\lambda<35 \mu \mathrm{m})$ to the total inte- grated flux; \\ - temperature of the black-body components.}

We assume that the less evolved the source, the colder, hence the SED peaks at longer wavelength with weaker flux. As the massive core evolves, it becomes warmer, thereby heating the 
dust wherein it is embedded. As a consequence, the contribution of the flux at shorter wavelength $\left(F_{35}\right.$, the integrated flux for $\lambda<35 \mu \mathrm{m}$ ) increases.

Applying these criteria, W43MM1 appears to be the youngest object. A sequence of evolution W43MM1 $\rightarrow$ IRAS18264-1152 $\rightarrow$ IRAS05358+3543 $\rightarrow$ IRAS18162-2048 is proposed and adopted for the following discussion. This evolutionary sequence is also suggested by the dynamical ages derived by the authors cited above.

\section{Observations}

The observations presented here were performed during two sessions. The first one in 2005 September used the IRAM$30 \mathrm{~m}$ antenna ${ }^{2}$. The second one in 2006 May used the Caltech Submillimeter Observatory ${ }^{3}$ on the summit of Mauna Kea (Hawaii).

With the 30m telescope, all sources were observed in the rotational transitions lines of CS $J=5-4$, SO $J=3_{4}-2_{3}$, $J=5_{6}-4_{5}, J=6_{5}-5_{4}, \mathrm{SO}_{2} J=5_{1,5}-4_{0,4}, J=10_{0,10}-9_{1,9}$, $J=11_{1,11}-10_{0,10}, J=14_{0,14}-13_{1,13}$, OCS $J=8-7, J=13-12$, $J=19-18, \mathrm{H}_{2} \mathrm{~S} J=1_{1,0}-1_{0,1}, J=2_{2,0}-2_{1,1}$, and in the isotopic lines of ${ }^{34} \mathrm{SO} J=3_{4}-2_{3}, \mathrm{SO}_{2} J=5_{1,5}-4_{0,4}, \mathrm{OC}^{34} \mathrm{~S} J=$ $8-7, \mathrm{H}_{2}^{34} \mathrm{~S} J=1_{1,0}-1_{0,1}$ (see Table 2, where are given for each energy level transition the observational parameters, i.e. half power beam width, main beam efficiency $\eta_{\mathrm{mb}}$, receiver name, velocity resolution $\delta v$ and system temperature $T_{\text {sys }}$ ). Only IRAS05358+3543, IRAS18264-1152, and W43MM1 were observed in the $\mathrm{CS}$ and $\mathrm{C}^{34} \mathrm{~S} J=3-2$ lines. Observations used multiple receivers (either simultaneously A100, B100, A230, $\mathrm{B} 230$, or $\mathrm{C} 150, \mathrm{D} 150, \mathrm{C} 270, \mathrm{D} 270)$ coupled to the highresolution VESPA backend (resolution of $80 \mathrm{kHz}$ ) in frequencyswitching model (frequency throw of $7.9 \mathrm{MHz}$ ). The sky conditions were reasonable $\left(\tau_{0}^{\text {atm }} \sim 0.1-0.5\right.$ at $225 \mathrm{GHz}$, see Table 2 for the $T_{\text {sys }}$ ). The telescope pointing (better than 2") and focus were set on suitable planets (i.e. Jupiter) or standard calibration sources.

Complementary observations were performed with the CSO telescope for lines with frequencies higher than $300 \mathrm{GHz}$ (to cover the energy ladder better). IRAS05358+3543 was not visible at the CSO during our run, so only the other three sources were observed in lines of CS $J=7-6$, SO $J=8_{8}-77, \mathrm{SO}_{2} J=$ $18_{0,18}-17_{1,17}$, and in corresponding isotopic lines of $\mathrm{C}^{34} \mathrm{~S},{ }^{34} \mathrm{SO}$, ${ }^{34} \mathrm{SO}_{2}$. The receiver is a helium-cooled SIS mixer operating in double-sideband mode designed to fully cover the $280-420 \mathrm{GHz}$ window. The backend consists of a 1024 channel acousto-optical spectrometer covering a bandwidth of $50 \mathrm{MHz}$, providing a velocity resolution of $\Delta v \simeq 0.086 \mathrm{~km} \mathrm{~s}^{-1}$ at $345 \mathrm{GHz}$. Observations were performed under good but unstable weather conditions $\left(\tau_{0}^{\text {atm }} \sim 0.06-0.15\right.$ at $225 \mathrm{GHz}$, see Table 2 for the $\left.T_{\text {sys }}\right)$. The pointing accuracy was about $4-5^{\prime \prime}$. The CS $J=7-6$ line was blended with an $\mathrm{H}_{2} \mathrm{CO}$ line from the image sideband; therefore, we applied a frequency shift to the local oscillator frequency to separate the two lines, but the procedure did not work well for IRAS18264-1152 and W43MM1 (so the CS spectra are contaminated).

Data reduction was performed using the CLASS software from the GILDAS suite (Guilloteau \& Lucas 2000). We found

\footnotetext{
2 http://iram.fr/IRAMES/index.htm

3 http://www.cso.caltech.edu. The Caltech Submillimeter Observatory is supported by the National Science Foundation under award AST-0540882.
}

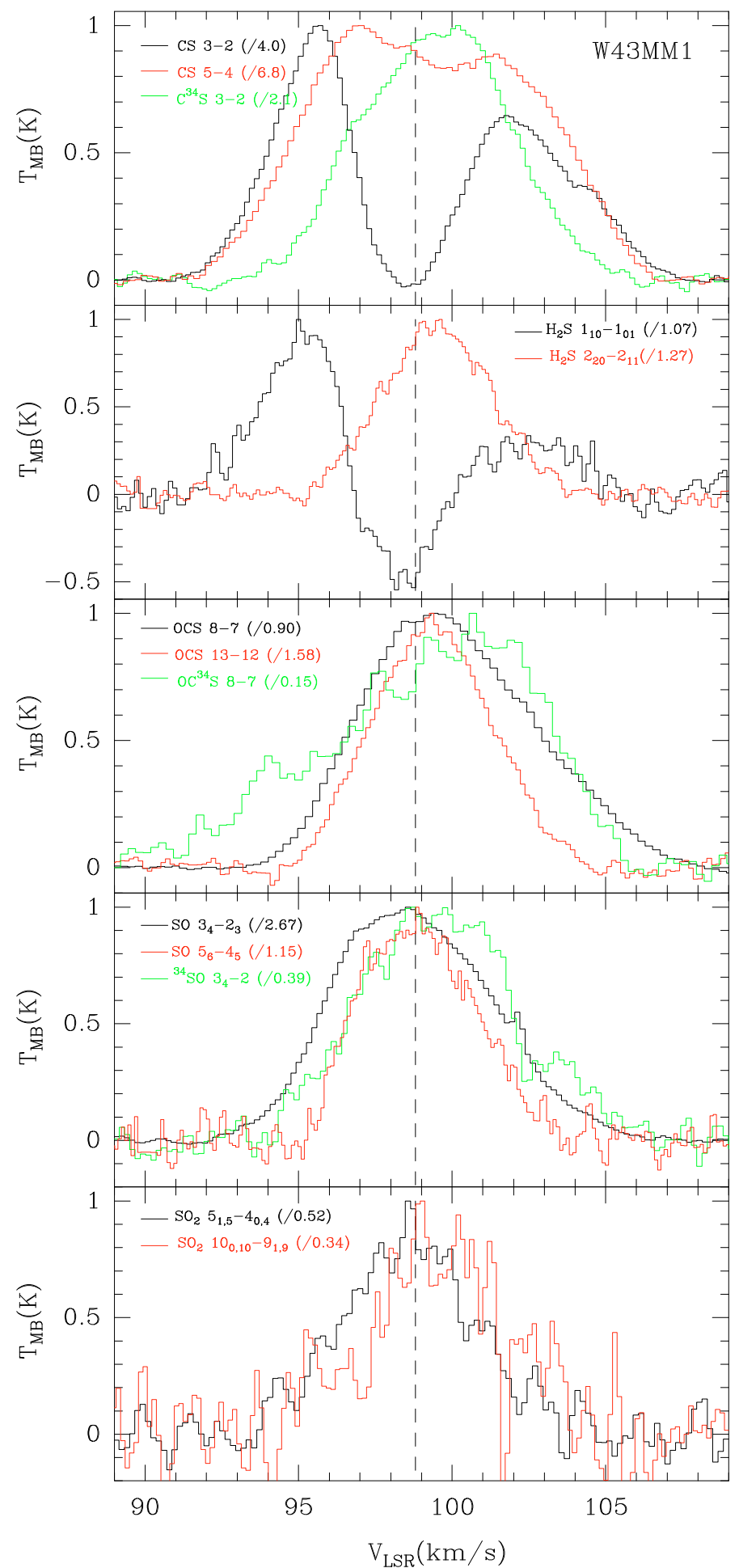

Fig. 2. Normalized emission of $\mathrm{CS}, \mathrm{H}_{2} \mathrm{~S}$, OCS, $\mathrm{SO}, \mathrm{SO}_{2}$ (and isotopic species) lines from W43MM1. Spectra velocity resolutions are 0.10 $0.19 \mathrm{~km} \mathrm{~s}^{-1}$. The dashed line shows the source LSR velocity.

and eliminated unusable data, subtracted a baseline, then spectra at the same position were summed, and finally antenna temperature $T_{\mathrm{a}}^{*}$ was converted into $T_{\mathrm{mb}}$ (using the $\eta_{\mathrm{mb}}$ values from Table 2). Special attention was paid to the frequency-switching observing method. 
Table 2. List of observational parameters for the IRAM 30m and CSO telescopes.

\begin{tabular}{|c|c|c|c|c|c|c|c|c|c|}
\hline Species & Line & $\begin{array}{c}\mathrm{E}_{\mathrm{up}} \\
{\left[\mathrm{cm}^{-1}\right]}\end{array}$ & $\begin{array}{c}\text { Frequency } \\
{[\mathrm{GHz}]}\end{array}$ & $\begin{array}{c}H P B W \\
{\left[{ }^{\prime \prime}\right]}\end{array}$ & Instrument $^{a}$ & $\eta_{\mathrm{mb}}$ & Receiver & $\begin{array}{c}\delta v \\
{\left[\mathrm{~m} \cdot \mathrm{s}^{-1}\right]}\end{array}$ & $\begin{array}{l}T_{\text {sys }} \\
{[\mathrm{K}]}\end{array}$ \\
\hline \multirow[t]{3}{*}{$\mathrm{CS}$} & $3-2$ & 10 & 146.969 & 17 & $30 \mathrm{~m}$ & 0.69 & $\bar{D} 150$ & 160 & $315-350$ \\
\hline & $5-4$ & 24 & 244.936 & 10 & $30 \mathrm{~m}$ & 0.48 & A230 & 96 & $530-860$ \\
\hline & $7-6$ & 46 & 342.883 & 24 & $\mathrm{CSO}$ & 0.75 & 345 & 43 & $1210-1340$ \\
\hline \multirow[t]{2}{*}{$\mathrm{C}^{34} \mathrm{~S}$} & $3-2$ & 10 & 144.617 & 17 & $30 \mathrm{~m}$ & 0.69 & D150 & 162 & $270-390$ \\
\hline & $7-6$ & 46 & 337.396 & 25 & $\mathrm{CSO}$ & 0.75 & 345 & 43 & $1260-1760$ \\
\hline \multirow[t]{4}{*}{ SO } & $3_{4}-2_{3}$ & 11 & 138.178 & 18 & $30 \mathrm{~m}$ & 0.70 & D150 & 170 & $300-330$ \\
\hline & $5_{6}-4_{5}$ & 24 & 219.949 & 11 & $30 \mathrm{~m}$ & 0.55 & B230 & 110 & 590-930 \\
\hline & $6_{5}-5_{4}$ & 35 & 251.825 & 10 & $30 \mathrm{~m}$ & 0.48 & D270 & 190 & $750-830$ \\
\hline & $8_{8}-7_{7}$ & 61 & 344.308 & 24 & $\mathrm{CSO}$ & 0.75 & 345 & 43 & 1020-1050 \\
\hline \multirow[t]{2}{*}{${ }^{34} \mathrm{SO}$} & $3_{4}-2_{3}$ & 11 & 135.775 & 18 & $30 \mathrm{~m}$ & 0.70 & D150 & 170 & $290-350$ \\
\hline & $8_{8}-7_{7}$ & 61 & 337.582 & 25 & $\mathrm{CSO}$ & 0.75 & 345 & 43 & $1020-1060$ \\
\hline \multirow[t]{6}{*}{$\mathrm{SO}_{2}$} & $5_{1,5}-4_{0,4}$ & 11 & 135.696 & 18 & $30 \mathrm{~m}$ & 0.70 & D150 & 170 & $270-400$ \\
\hline & $10_{0,10}-9_{1,9}$ & 34 & 160.827 & 15 & $30 \mathrm{~m}$ & 0.66 & C150 & 150 & $350-520$ \\
\hline & $11_{1,11}-10_{0,10}$ & 42 & 221.965 & 11 & $30 \mathrm{~m}$ & 0.55 & B230 & 100 & $530-550$ \\
\hline & $14_{0,14}-13_{1,13}$ & 65 & 244.254 & 10 & $30 \mathrm{~m}$ & 0.48 & A230 & 190 & $830-1430$ \\
\hline & $18_{0,18}-17_{1,17}$ & 105 & 321.330 & 26 & $\mathrm{CSO}$ & 0.75 & 345 & 45 & $770-820$ \\
\hline & $28_{4,24}-28_{3,25}$ & 289 & 267.719 & 9 & $30 \mathrm{~m}$ & 0.46 & D270 & 170 & $470-510$ \\
\hline${ }^{34} \mathrm{SO}_{2}$ & $5_{1,5}-4_{0,4}$ & 11 & 133.471 & 18 & $30 \mathrm{~m}$ & 0.70 & D150 & 170 & $200-220$ \\
\hline \multirow[t]{3}{*}{$\mathrm{OCS}$} & $8-7$ & 14 & 97.301 & 25 & $30 \mathrm{~m}$ & 0.77 & A100 & 240 & $140-160$ \\
\hline & $13-12$ & 37 & 158.107 & 15 & $30 \mathrm{~m}$ & 0.66 & C150 & 150 & $340-360$ \\
\hline & $19-18$ & 77 & 231.060 & 11 & $30 \mathrm{~m}$ & 0.52 & A230 & 100 & $450-580$ \\
\hline $\mathrm{OC}^{34} \mathrm{~S}$ & $8-7$ & 14 & 94.922 & 25 & $30 \mathrm{~m}$ & 0.77 & B100 & 240 & $140-160$ \\
\hline \multirow[t]{2}{*}{$\mathrm{H}_{2} \mathrm{~S}$} & $1_{1,0}-1_{0,1}$ & 19 & 168.762 & 14 & $30 \mathrm{~m}$ & 0.70 & D150 & 140 & $610-800$ \\
\hline & $2_{2,0}-2_{1,1}$ & 58 & 216.710 & 11 & $30 \mathrm{~m}$ & 0.57 & B230 & 110 & $410-600$ \\
\hline $\mathrm{H}_{2}^{34} \mathrm{~S}$ & $1_{1,0}-1_{0,1}$ & 19 & 167.910 & 14 & $30 \mathrm{~m}$ & 0.70 & D150 & 140 & $630-670$ \\
\hline
\end{tabular}

${ }^{a}$ Conversional factor is $S / T_{\mathrm{mb}}=4.95 \mathrm{Jy} / \mathrm{K}$ for IRAM $30 \mathrm{~m}$ telescope and $S / T_{\mathrm{mb}}=74 \mathrm{Jy} / \mathrm{K}$ for CSO telescope.

\section{Results}

We present here the results of the observations for each source. Some spectra of the detected lines are displayed in Figs. 2-5 (the remaining spectra are available as online material). Some transitions $\left({ }^{34} \mathrm{SO} 8_{8}-77, \mathrm{SO}_{2} 28_{4,24}-28_{3,25}\right.$, and $\left.{ }^{34} \mathrm{SO}_{2} 5_{1,5}-4_{0,4}\right)$ are not detected in any of our sources. The line parameters are given in Tables 6-9 (only available in electronic form): linewidth at $3 \sigma$ level $\Delta v_{3 \sigma}$, half power linewidth $\Delta v_{1 / 2}$, and the line flux derived from Gaussian fits, with several components, made with CLASS software; for the non detection, $1 \sigma$ upper limits are given. For all observations, contamination from lines in the image sideband of the receiver has been checked.

\subsection{W43MM1}

The CS line emission from this source is characterized by what looks like a strong self-absorption (at $\sim-0.2 \mathrm{~km} \mathrm{~s}^{-1}$ from the source velocity) with a stronger blue component compared to the red one. Indeed the self-absorption in the 3-2 line goes below zero kelvins, splitting the line profile into two well-separated components. The $2 \mathrm{~mm}$ continuum flux is actually high $(0.8 \mathrm{Jy})$, which converts into a brightness temperature of $5.5 \mathrm{~K}$, and can therefore explain the observed dip. Similar, but weaker, selfabsorption is observed in the 5-4 and 7-6 transitions. However, the isotopic lines are only weakly asymmetric, redshifted with respect to the systemic velocity. The derived opacities (from our model, see Sect. 6.2) are 0.4 and 0.6 for the $\mathrm{C}^{34} \mathrm{~S} 3-2$ and 7-6 line emissions, respectively, indicating optically thick lines. All lines are very broad, up to $15 \mathrm{~km} \mathrm{~s}^{-1}$. The $\mathrm{HCO}^{+}$and $\mathrm{H}^{13} \mathrm{CO}^{+} 3-2$ emission lines observed by Motte et al. (2003) have exactly the same profiles as the $\mathrm{CS}$ and $\mathrm{C}^{34} \mathrm{~S}$ emission lines, respectively. The self-absorption is present in the $\mathrm{H}_{2} \mathrm{~S}$ line emissions, too (the isotopic line is not detected), even producing a negative dip for the $1_{1,0}-1_{0,1}$ line, below the continuum level, at the same velocity as CS. The $2_{2,0}-2_{1,1}$ line emission has a different profile with more red-shifted emission.

The SO $3_{4}-2_{3}, 5_{6}-4_{5}$ and the isotopic $3_{4}-2_{3}$ line profiles are also weakly asymmetric, with a more pronounced blueshifted emission. The $6_{5}-5_{4}$ and $8_{8}-7_{7}$ lines exhibit, on the contrary, a stronger redshifted component. The $\mathrm{SO}_{2} 5_{1,5}-4_{0,4}$ line is also blue-asymmetric, but all other $\mathrm{SO}_{2}$ lines are more or less symmetrical and Gaussian.

All OCS lines exhibit a Gaussian profile, slightly red-shifted by $\sim 0.5 \mathrm{~km} \mathrm{~s}^{-1}$ from the source velocity, with a red wing. The isotopic $\mathrm{OC}^{34} \mathrm{~S}$ line profile is complicated by several components in emission at 91.8, 94.0, and $95.5 \mathrm{~km} \mathrm{~s}^{-1}$ (see Fig. 2). No identification with known species was possible (we also checked the frequencies in the image band) and instrumental effects have been ruled out. This weak component is not seen in the corresponding OCS line profile. Since W43MM1 is a very rich molecular source (see Sect. 7.1), this could well be real lines.

\subsection{IRAS18264-1152}

All line emission profiles peaked on the source velocity and have more or less the same shape, with a blue wing. Nonetheless, a distinct weak emission at $48-50 \mathrm{~km} \mathrm{~s}^{-1}$ is observed for all transitions of all species. These emissions cannot be due to a contamination from any OFF position as observations were performed in frequency-switch mode. None of these features correspond to a known molecular line. We propose that this secondary emission comes from a different location than the main one, either from another source within the beam, or from a knot in the outflow. Actually, an $\mathrm{SiO}$ outflow component at $48 \mathrm{~km} \mathrm{~s}^{-1}$, offset by $20^{\prime \prime}$ has been observed by Qiu et al. (2007). 


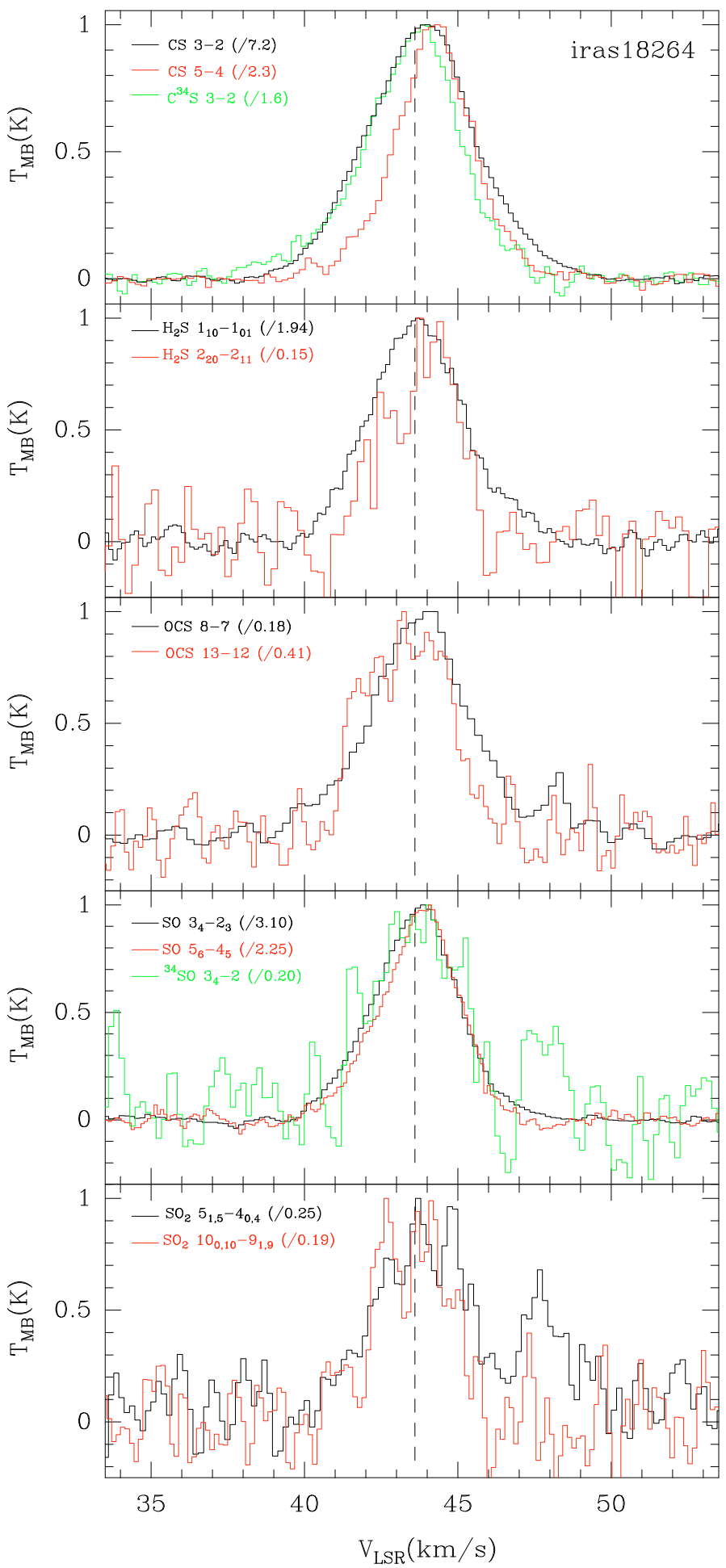

Fig. 3. Normalized emission of $\mathrm{CS}, \mathrm{H}_{2} \mathrm{~S}$, OCS, $\mathrm{SO}, \mathrm{SO}_{2}$ (and isotopic species) lines from IRAS18264-1152. Spectra velocity resolutions are $0.10-0.19 \mathrm{~km} \mathrm{~s}^{-1}$. The dashed line shows the source LSR velocity.

All CS lines are detected, except the 7-6 transition that is blended with an $\mathrm{H}_{2} \mathrm{CO}$ line from the image band, hence completely contaminating the profile. The $C^{34} S 7-6$ line is tentatively detected. The $\mathrm{H}_{2} \mathrm{~S}$ lines exhibit a less peaked profile with a blue wing, too. All the SO lines are detected too, but the $65_{5}-5_{4}$ line emission exhibits a strong and very broad red wing that looks suspicious. We do not consider this line for further analysis. The $\mathrm{SO}_{2}$ line profiles are more flattened. Actually, an

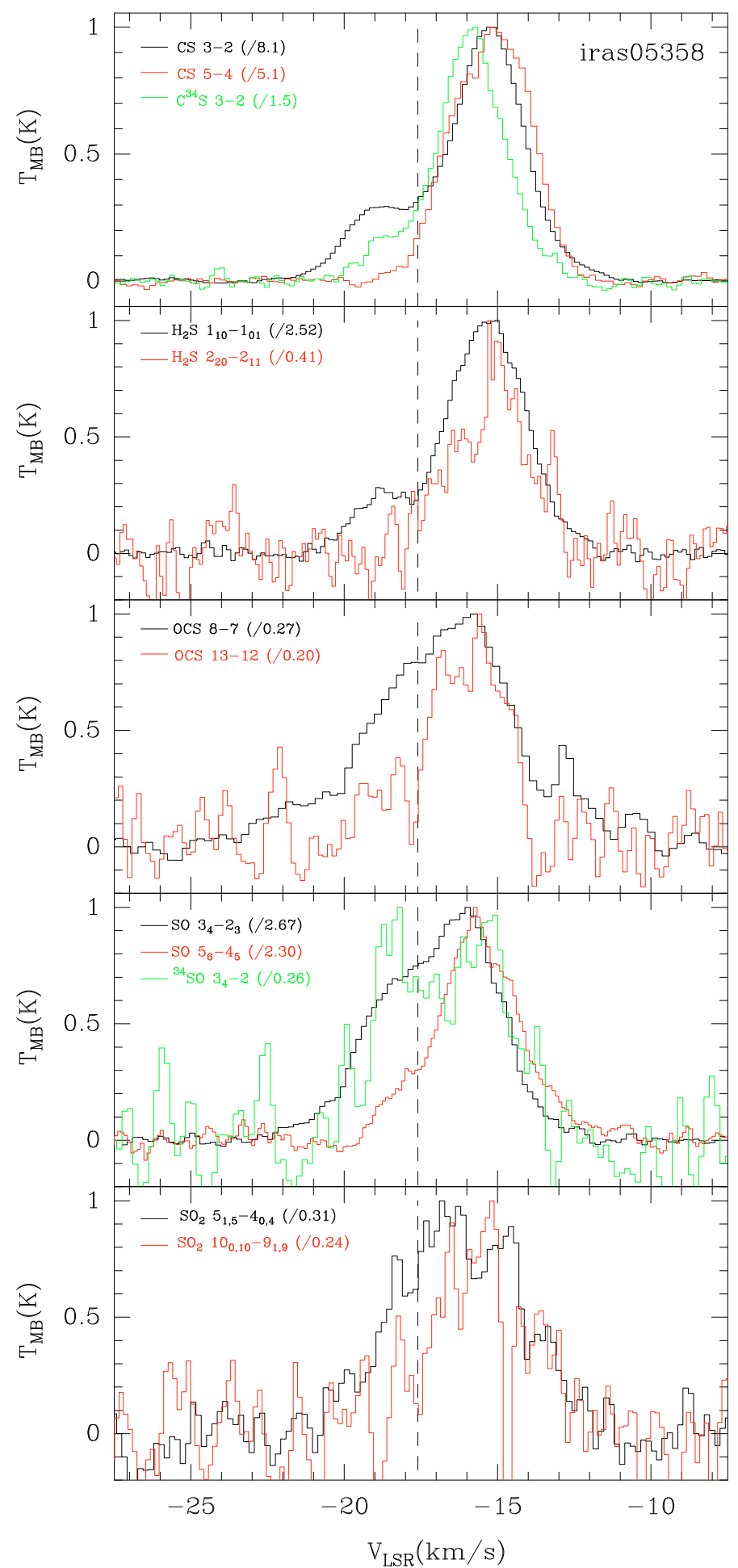

Fig. 4. Normalized emission of $\mathrm{CS}, \mathrm{H}_{2} \mathrm{~S}$, OCS, SO, $\mathrm{SO}_{2}$ (and isotopic species) lines from IRAS05358+3543. Spectra velocity resolutions are $0.10-0.19 \mathrm{~km} \mathrm{~s}^{-1}$. The dashed line shows the source LSR velocity.

unidentified line at $47.8 \mathrm{~km} \mathrm{~s}^{-1}$ is observed in the $\mathrm{SO}_{2} 5_{1,5}-4_{0,4}$ spectra, and could come from the reference OFF spectra, just as the suspicious $6_{5}-5_{4}$ wing. The OCS lines exhibit similar profiles, with a blue asymmetry.

\subsection{IRAS05358+3543}

The CS line emission is strong in both observed transitions (the 3-2 line emission being the strongest and broadest one) with 


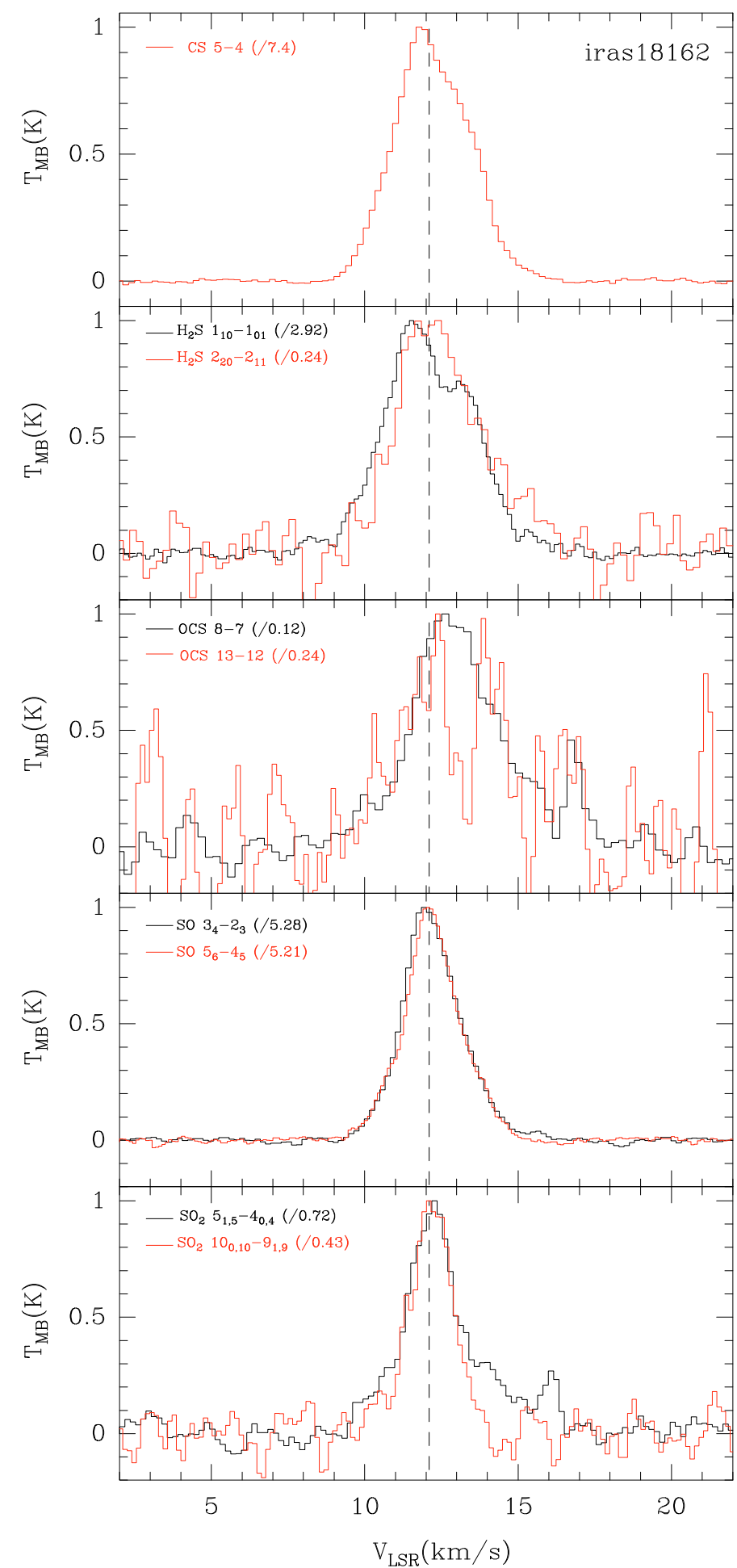

Fig. 5. Normalized emission of $\mathrm{CS}, \mathrm{H}_{2} \mathrm{~S}$, OCS, $\mathrm{SO}, \mathrm{SO}_{2}$ (and isotopic species) lines from IRAS18162-2048. Spectra velocity resolutions are $0.10-0.19 \mathrm{~km} \mathrm{~s}^{-1}$. The dashed line shows the source LSR velocity.

a strong dip at the source velocity, even for the isotopic line. The isotopic line emission here is optically thin (around 0.1 according to our model), therefore this dip likely stems from two velocity components rather than to self-absorption. All profiles are asymmetrical with a stronger red component, the blue one being almost completely extinguished (nearly in absorption) for the CS 5-4 line emission. The peak-to-peak velocity separation is $3.0,3.5$, and $4.3 \mathrm{~km} \mathrm{~s}^{-1}$ for the $\mathrm{C}^{34} \mathrm{~S} 3-2, \mathrm{CS} 3-2$ and 5-4 lines, respectively.

The $\mathrm{H}_{2} \mathrm{~S} 1_{1,0}-1_{0,1}$ line emission also exhibits a strong dip at the source velocity. The separation between the two emission peaks is $3.6 \mathrm{~km} \mathrm{~s}^{-1}$. The same shape is also seen for the $2,{ }_{2,0}-2_{1,1}$ line, where no blue peak is seen, with the red component split into two peaks. The isotopic line might be tentatively detected, with very strong asymmetry.

Profiles of the SO lines generally exhibit a dip at the source velocity. The peak-to-peak separation is $3 \mathrm{~km} \mathrm{~s}^{-1}$ for the $3_{4}-2_{3}$ line. The isotopic $3_{4}-2_{3}$ line shows a red-shifted (by more than $1 \mathrm{~km} \mathrm{~s}^{-1}$ ) double-horn profile. ${ }^{34} \mathrm{SO} 3_{4}-2_{3}$ line emission is marginally detected, suffering from obvious baseline problems. We checked, without success, if the double-peak profile could be due to other species.

The $\mathrm{SO}_{2}$ emission is detected in all transitions (except the $11_{1,11}-10_{0,10}$ ), but the $S / N$ is quite low (5-9). All the lines are asymmetric, nearly without any red component. The $14_{0,14}-13_{1,13}$ emission is the strongest. A dip is seen in all OCS lines too, most prominently in the 13-12 transition. The 1918 line is the strongest. The OCS $13-12$ line is only tentatively detected with a complex profile.

A second emission component is seen in the $\mathrm{C}^{34} \mathrm{~S} 3-2$, OCS $8-7$, SO $3_{4}-2_{3}, \mathrm{SO}_{2} 5_{1,5}-4_{0,4}$ spectra at $-13 \mathrm{~km} \mathrm{~s}^{-1}$. As this emission is also present in the wing of the CS 3-2 and $\mathrm{H}_{2} \mathrm{~S} 1-$ 1 line emission (observed with a smaller telescope beam), we believe that this emission originates from the source $\mathrm{mm} 1 \mathrm{~b}$ as observed in the $C^{34}$ S 5-4 line emission by Leurini et al. (2007) at the same velocity.

\subsection{IRAS18162-2048}

The CS line shapes are similar for the different transitions observed, with the 5-4 line substantially stronger and broader, exhibiting a red wing. The $\mathrm{H}_{2} \mathrm{~S}$ emission is slightly broader than $\mathrm{CS}$, with more complex profiles: the $1_{1,0}-1_{0,1}$ emission profile is made of two peaks, possibly revealing self-absorption at a redshifted velocity (by $0.5 \mathrm{~km} \mathrm{~s}^{-1}$ with respect to the source systemic velocity). The $2_{2,0}-2_{1,1}$ line profile is slightly asymmetrical, with a red wing. The optically thin isotopic line emission exhibits a symmetrical profile centered on the source velocity, with a narrower profile than for the $\mathrm{H}_{2} \mathrm{~S}$ emission.

The SO lines are strong and easily detected, but the isotopic line is not detected. The $\mathrm{SO}_{2}$ lines are clearly detected, even the $18_{0,18}-17_{1,17}$ transition, and the $11_{1,11}-10_{0,10}$ is the strongest one. The line profiles show a red wing.

All the OCS lines are completely asymmetrical with a very weak blue component, indicating a self-absorption at the source velocity. For that source, too, emission a few $\mathrm{km} \mathrm{s}^{-1}$ away from the main emission is detected (at $16-17 \mathrm{~km} \mathrm{~s}^{-1}$ ) for the lines observed with a beam larger than $18^{\prime \prime}$, likely revealing the presence of a second source within the beam.

\section{Analysis}

\subsection{Line asymmetries}

Analysis of the line profiles can reveal the presence of outflows, infall or even rotation because of their known signatures (see Fuller et al. 2005, and references therein): outflow or rotation give rise to both red and blue asymmetric lines, while infall produces only blue ones. Indeed, the profiles of optically thick lines from infalling material have stronger blueshifted emission than does the redshifted one. However, it is important to stress that 
Table 3. Asymmetry parameter $\delta v$ (Mardones et al. 1997) calculated for the detected line emissions.

\begin{tabular}{lccccc}
\hline \hline Species & Line & \multicolumn{5}{c}{$\delta v$} \\
& & W43MM1 & 18264 & 05358 & 18162 \\
\hline $\mathrm{CS}$ & $3-2$ & -0.69 & 0.05 & 0.37 & \\
& $5-4$ & -0.41 & 0.16 & 0.42 & 0.03 \\
& $7-6$ & -0.40 & $a$ & & -0.01 \\
$\mathrm{C}^{34} \mathrm{~S}$ & $3-2$ & 0.05 & -0.02 & 0.27 & \\
& $7-6$ & 0.18 & 0.20 & & 0.13 \\
$\mathrm{SO}$ & $3_{4}-2_{3}$ & -0.10 & 0.00 & 0.22 & 0.03 \\
& $5_{6}-4_{5}$ & -0.06 & 0.05 & 0.29 & 0.06 \\
& $6_{5}-5_{4}$ & 0.11 & $b$ & 0.28 & -0.08 \\
& $8_{8}-7_{7}$ & 0.11 & 0.04 & & 0.12 \\
& $5_{1,5}-4_{0,4}$ & -0.11 & 0.08 & 0.17 & 0.03 \\
$\mathrm{SO}_{2}$ & $10_{0,10}-9_{1,9}$ & 0.05 & -0.02 & 0.27 & 0.05 \\
& $11_{1,11}-10_{0,10}$ & & -0.03 & & 0.44 \\
& $14_{0,14}-13_{1,13}$ & & -0.12 & 0.38 & 0.00 \\
& $18_{0,18}-17_{1,17}$ & 0.10 & & & 0.19 \\
$\mathrm{OCS}^{2}$ & $8-7$ & 0.11 & 0.06 & 0.26 & 0.53 \\
& $13-12$ & 0.03 & -0.06 & 0.24 & 0.52 \\
& $19-18$ & 0.10 & 0.09 & 0.17 & 1.08 \\
$\mathrm{H}_{2} \mathrm{~S}$ & $1_{1,0}-1_{0,1}$ & -0.76 & 0.02 & 0.39 & -0.37 \\
& $2_{2,0}-2_{1,1}$ & 0.06 & 0.08 & 0.42 & 0.16 \\
\hline
\end{tabular}

${ }^{a}$ Doubtful line; ${ }^{b}$ doubtful line.

outflow or rotation could also produce a blue asymmetric line profile along a particular line of sight to a source.

To quantify the asymmetry of a line, we use Mardones et al. (1997) criteria, by calculating the asymmetry parameter

$\delta v=\frac{v_{\text {thick }}-v_{\text {thin }}}{\Delta v_{1 / 2}}$

where $v_{\text {thick }}$ and $v_{\text {thin }}$ are the line peak velocity of an optically thick and an optically thin tracer, respectively, and $\Delta v_{1 / 2}$ the line width $(F W H M)$ of the optically thin line.

Because not all the same lines are detected or observed in all sources, the difficulty is to find a common strong optically thin line, generally from isotopic species. We use ${ }^{34} \mathrm{SO}_{4}-2_{3}$ for IRAS05358+3543 $\left(v_{\text {thin }}=-17.0 \mathrm{~km} \mathrm{~s}^{-1}, \Delta v_{1 / 2}=4.4 \mathrm{~km} \mathrm{~s}^{-1}\right)$, IRAS18264-1152 $\left(v_{\text {thin }}=43.6 \mathrm{~km} \mathrm{~s}^{-1}, \Delta v_{1 / 2}=4.0 \mathrm{~km} \mathrm{~s}^{-1}\right)$, W43MM1 $\left(v_{\text {thin }}=99.2 \mathrm{~km} \mathrm{~s}^{-1}, \Delta v_{1 / 2}=5.5 \mathrm{~km} \mathrm{~s}^{-1}\right)$, and $\mathrm{H}_{2}{ }^{34} \mathrm{~S}_{1,0}-1_{0,1}$ for IRAS18162-2048 $\left(v_{\text {thin }}=12.1 \mathrm{~km} \mathrm{~s}^{-1}\right.$, $\Delta v_{1 / 2}=1.5 \mathrm{~km} \mathrm{~s}^{-1}$ ). Results are given in Table 3 .

Negative values of $\delta v$ correspond to blueshifted emission and positive values to a redshifted one. Following Mardones et al. (1997) and Fuller et al. (2005), we adopt a criteria of $|\delta v|>0.25$ to indicate that a line profile is asymmetric. Main conclusions are that the source IRAS18264-1152 has symmetric lines, except for the $\mathrm{C}^{34} \mathrm{~S} 7-6$ line (close to the 0.25 value), while for IRAS18162-2048 asymmetric lines are observed in OCS, in the $\mathrm{H}_{2} \mathrm{~S}_{1,0}-1_{0,1}$ (redshifted) and in the $\mathrm{SO}_{2}$ 11-10 transitions (blueshifted). Very strong asymmetries are observed for the two other sources: most of the lines from IRAS05358+3543 are strongly redshifted ( $\delta v$ up to 0.42 ); W43MM1 shows clearly blueshifted lines in CS (up to -0.69 ) and $\mathrm{H}_{2} \mathrm{~S}$ (up to -0.76 ), probably indicating the presence of infall towards this source. Nevertheless, we stress again that such an emission profile can be sometimes produced by an outflow too, e.g. CO 1-0 line profile towards IRAS18162-2048 by Benedettini et al. (2004). As a result, this option cannot be eliminated without further investigations.
Table 4. Summary of results from dust continuum emission modeling (above mid-line) and from molecular line emission modeling (below mid-line).

\begin{tabular}{ccccc}
\hline \hline Source & W43MM1 $^{a}$ & 18264 & 05358 & 18162 \\
\hline$d(\mathrm{kpc})$ & 5.5 & 3.5 & 1.8 & 1.9 \\
$L\left(10^{4} L_{\odot}\right)$ & 2.3 & 1.4 & 0.7 & 2.9 \\
$T_{*}\left(10^{4} \mathrm{~K}\right)$ & 3.0 & 2.9 & 2.6 & 3.0 \\
$r_{\text {Out }}\left(10^{4} \mathrm{AU}\right)$ & 2.5 & 2.7 & 2.1 & 2.6 \\
$p^{b}$ & -2.0 & -1.5 & -1.4 & -2.1 \\
$n_{0}{ }^{c}\left(10^{4}\right)$ & 40 & 1.0 & 0.3 & 6.1 \\
$r_{\text {sub }}(\mathrm{AU})$ & 4.9 & 26.5 & 20.1 & 27.2 \\
$\alpha$ & -0.54 & -0.61 & -0.62 & -0.60 \\
$\beta$ & 3.55 & 4.05 & 3.99 & 4.04 \\
$\langle T\rangle(\mathrm{K})$ & 20.0 & 32.6 & 31.1 & 35.6 \\
$T_{\text {out }}(\mathrm{K})$ & 14.2 & 24.6 & 24.1 & 27.2 \\
$\langle n\rangle\left(10^{6} \mathrm{~cm}^{-3}\right)$ & 11.8 & 2.6 & 0.2 & 1 \\
$M\left(M_{\odot}\right)$ & 4100 & 1200 & 400 & 570 \\
$v_{\mathrm{t}}\left(\mathrm{km} \mathrm{s}^{-1}\right)$ & $0.8-1.5$ & $0.7-1.3$ & $0.7-2.6$ & $0.5-1.8$ \\
$v_{\text {infall }}\left(\mathrm{km} \mathrm{s}^{-1}\right)$ & $-(3.5-0.5)$ & & & \\
\hline
\end{tabular}

${ }^{a}$ Source description from Motte et al. (2003); ${ }^{b}$ from Beuther et al. (2002), excepted for $\mathrm{W} 43 \mathrm{MM} 1 ;{ }^{c}$ density at $100 \mathrm{AU} ;{ }^{d} \log (T)=$ $\alpha \log (r)+\beta$.

\subsection{Possible infall in W43MM1}

Assuming the observed CS profile towards W43MM1 is due to infall, we estimate the infall velocity using the approximate method described in Myers et al. (1996) for the optically thick lines with a strong blue asymmetry:

$v_{\text {in }} \approx \frac{\sigma^{2}}{v_{\text {red }}-v_{\text {blue }}} \ln \left(\frac{1+e T_{\mathrm{BD}} / T_{\mathrm{D}}}{1+e T_{\mathrm{RD}} / T_{\mathrm{D}}}\right)$,

where $T_{\mathrm{D}}$ is the brightness temperature of the dip (assumed optically thick), $T_{\mathrm{BD}}$ and $T_{\mathrm{RD}}$ the height of the blue and red peaks above the dip, respectively, and $v_{\text {blue }}$ and $v_{\text {red }}$ the velocity of the blue and red peaks, respectively. The velocity dispersion $\sigma$ is obtained from the $F W H M$ of an optically thin line.

By adopting the ${ }^{34} \mathrm{SO} 3_{4}-23$ line as an optically thin tracer, the velocity dispersion in the circumstellar material is estimated to be $5.5 \mathrm{~km} \mathrm{~s}^{-1}$ and the source velocity to be $99.2 \mathrm{~km} \mathrm{~s}^{-1}$. The derived infall velocities $v_{\text {in }}$ are $2.1,1.8$ and $2.9 \mathrm{~km} \mathrm{~s}^{-1}$, calculated for the CS 3-2, 5-4, and $\mathrm{H}_{2} \mathrm{~S}_{1,0}-1_{0,1}$ line emissions, respectively. Using the following formula (where $m$ is the mean mass of the molecule and $r_{\text {out }}$ the inner radius of the most outer layer):

$\frac{\mathrm{d} M}{\mathrm{~d} t}=4 \pi r_{\text {out }}^{2} m n v_{\text {in }}$,

where the inferred kinematic mass infall rate (for a size $r_{\text {out }}$ of $3.4 \times 10^{17} \mathrm{~cm}$ and a density $n$ of $2.5 \times 10^{6} \mathrm{~cm}^{-3}$, cf. Table 4 ) is $5.8-9.4 \times 10^{-2} M_{\odot} / \mathrm{yr}$.

\subsection{Outflows in the other sources or multiplicity effect?}

The $\mathrm{SO}_{4}-22_{3}$ lines towards IRAS18162-2048 can be fitted with two Gaussians of $F W H M$ line widths of 1.2 and $2.6 \mathrm{~km} \mathrm{~s}^{-1}$ (see Fig. 6). The CS 7-6 line emission can be fitted by two Gaussians too: one of $1 \mathrm{~km} \mathrm{~s}^{-1}$ broad, the other one of $2.8 \mathrm{~km} \mathrm{~s}^{-1}$. The optically thin $\mathrm{H}_{2}{ }^{34} \mathrm{~S}$ line can be fitted by two Gaussians of FHWM 1.2 and $3.2 \mathrm{~km} \mathrm{~s}^{-1}$, comparable to the components derived from the other lines. Moreover, it is interesting to note that the two Gaussian components are centered on velocities (11.9 and $12.3 \mathrm{~km} \mathrm{~s}^{-1}$ ) comparable to the velocities (11.9 and 

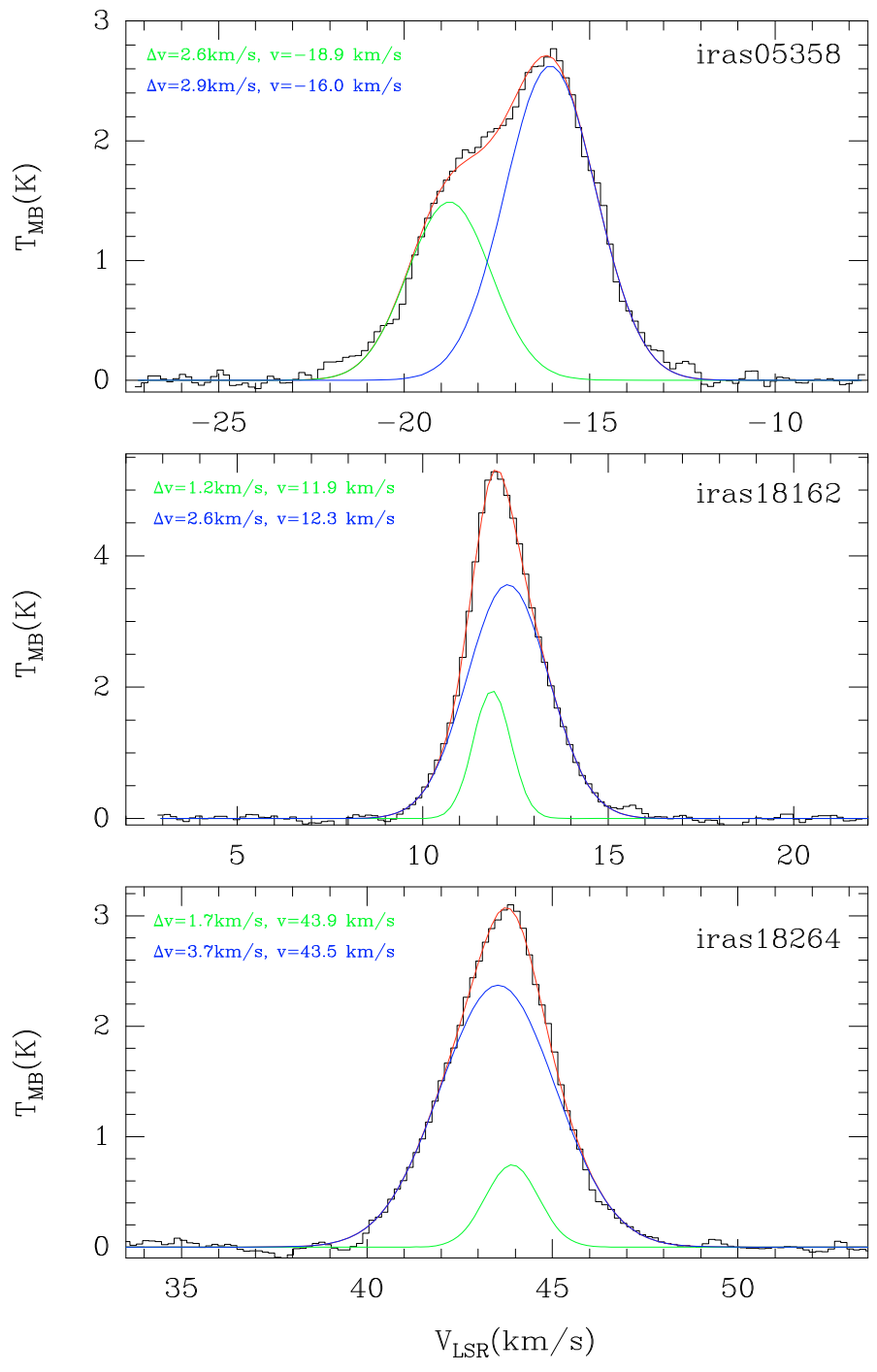

Fig. 6. Gaussian fit of the $\mathrm{SO}_{4}-2_{3}$ line emission from IRAS05358+3543, IRAS18162-2048 and IRAS18264-1152. The observed spectra are in black, total fit in red, individual components in blue and green.

$12.8 \mathrm{~km} \mathrm{~s}^{-1}$ ) of the two major outflow components seen in $\mathrm{CO}$, ${ }^{13} \mathrm{CO}$ and $\mathrm{C}^{18} \mathrm{O} 1-0$ line emissions by Benedettini et al. (2004). For IRAS181264, the CS $3-2$ and SO $3_{4}-2$ line emissions can be fitted with two Gaussians of two different FWHM: 1.7 and $3.7 \mathrm{~km} \mathrm{~s}^{-1}$. These two-components reveal the presence of gas flows, possibly an outflow towards objects IRAS18162-2048 and IRAS18264-1152.

Line emission profiles seen in the source IRAS05358+3543 exhibit a strong dip (especially for CS), even for the optically thin isotopic species, which is thus not a self-absorption but rather a the signature of two well-separated velocity components with $F W H M$ around 2.6 and $2.9 \mathrm{~km} \mathrm{~s}^{-1}$ (derived from CS 3-2 and $\mathrm{SO}_{4}-2{ }_{3}$ lines emission fitting, see Fig. 6). This could be due to the outflow detected by Beuther et al. (2002a) and Beuther et al. (2002c) or to the multiplicity of the source itself. IRAS05358 +3543 is indeed composed of three mm sources (Leurini et al. 2007), all within our beam, mm1 being the most massive, the other two being less evolved (mm3 is probably a starless massive core). Actually, the component at $-16 \mathrm{~km} \mathrm{~s}^{-1}$ is at the same velocity as the source mm2 (Leurini et al. 2007); hence, the profile may result from the source multiplicity.

\section{Modeling the continuum and the molecular emission of the sources}

We use the modeling method described in Marseille et al. (2008) and originally developed by Hogerheijde \& van der Tak (2000). It consists, in a first step, of constraining the physical structure of the source using a modeling of the SED, and in a second step of applying the obtained physical model to derive abundances of the observed species. To reduce the number of free parameters, we restrict our modeling to a simple 1D, radial description of the sources. The assumed spherical symmetry of the sources is acceptable if we restrict our study to the molecular line emission modeling coming from cold gas $(T<100 \mathrm{~K})$ as Marseille et al. (2008) show it for the massive dense core case.

\subsection{SED distribution}

We use the radiative transfer code MC3D (Wolf et al. 1999) in its $1 \mathrm{D}$ version to model the SED. The model parameters are taken from the literature (distance, $d$, power law index, $p$, for density distribution, heating source temperature, $T_{*}$, and source sizes, $r_{\text {out }}$, the total luminosity, $L$ ). The parameter, $p$, which defines the density distribution through a power law in the form $\rho(r) \propto r^{p}$, was derived by Beuther et al. (2002c) and Motte et al. (2003) from fits to the millimeter continuum emission maps. Concerning the source size, we systematically adopt the size of the continuum mm-wave emission, deconvolved by the beam. These values are summarized in Table 4 . The actual value used for the inner radius of the physical structure has virtually no observable effect on the results, so we adopt the radius of dust sublimation taken at $1500 \mathrm{~K}$ for the inner radius $\left(r_{\text {sub }}\right)$.

The fit of the millimeter and submillimeter SED of each source allows deriving the best temperature and distributions by iterating on the total mass, which is controlled by the value of the density $n_{0}$ at reference radius $r_{0}$ taken equal to $100 \mathrm{AU}$, and on the average temperature. The values obtained, with mean densities and temperatures of the sources, are reported in Table 4 and shown in Fig. 7.

\subsection{Modeling of the molecular emission}

The physical description of the sources obtained from the SED modeling is then used to model the molecular emission. We use the RATRAN radiative transfer code developed by Hogerheijde \& van der Tak (2000). We build data cubes with a velocity resolution equivalent to the observations, and with a high spatial resolution $\left(0.5^{\prime \prime}\right)$, which are convolved with the beam sizes of the observations for a direct comparison with the observed spectra. We adopt constant abundances for the RATRAN modeling. The abundances of all species are derived independently for each observed transitions of each species. Then the different abundances obtained for each particular species are interpreted as due to change in abundance as a function of the excitation temperature and therefore as a function of depth into the sources.

The line profiles observed are also affected by gas motions, particularly through the turbulence that is mainly responsible for the line emission widths seen in massive protostars. Thus we add a turbulent velocity field characterized by an rms velocity $v_{\mathrm{T}}$, for each transition of each molecular species (actually OCS lines are the most sensitive to the gas components in our model). In 

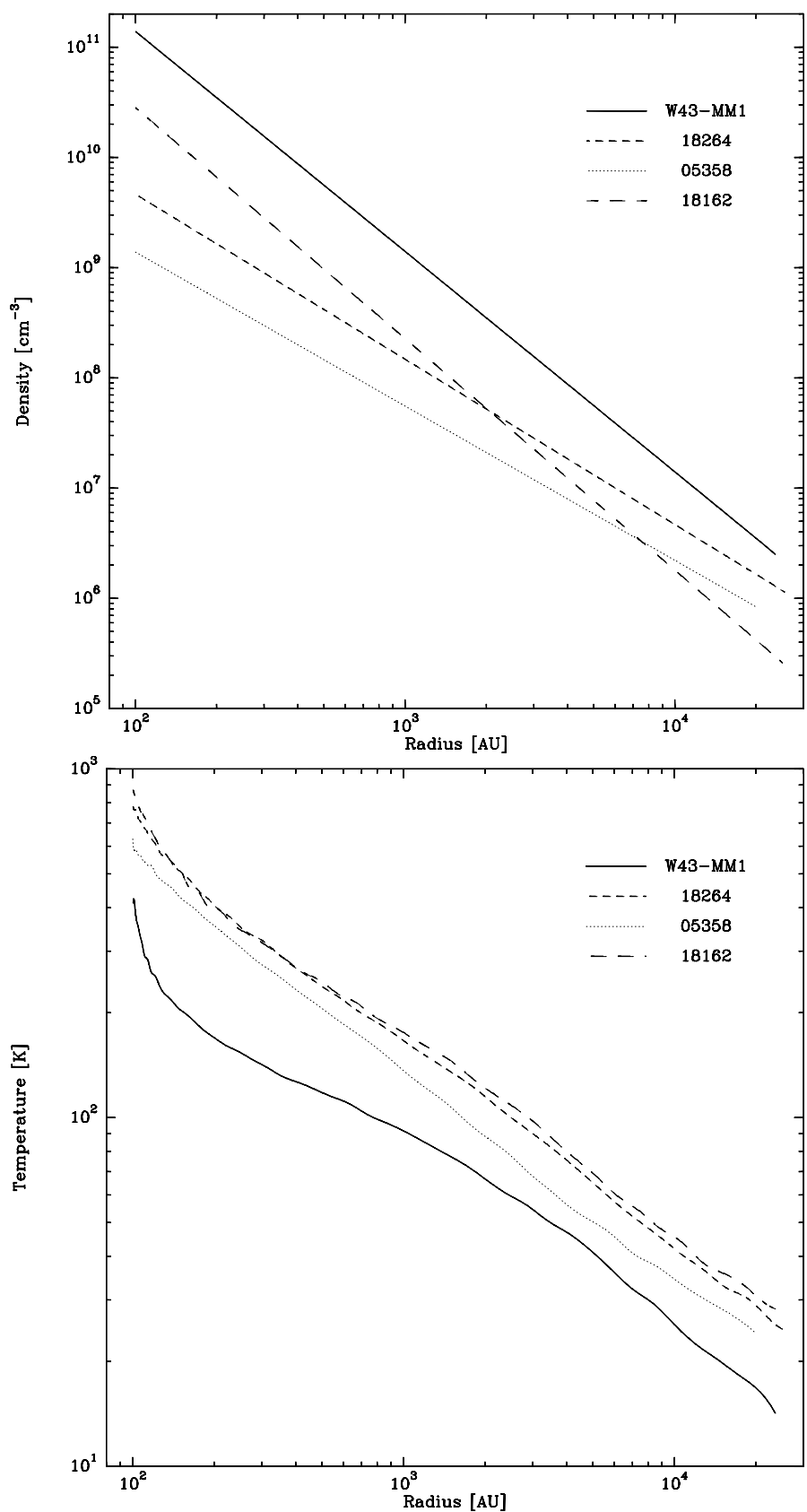

Fig. 7. Plot of the density (top) and temperature (bottom) versus the distance to the center for the 4 objects.

the case of W43MM1, where asymmetric profiles possibly due to infall are detected, we introduce a radial velocity field that follows a law in the form $v_{\text {inf }}=v_{0} / \sqrt{r / r_{\text {out }}}$, and fit the asymmetry parameter with different values of $v_{0}$ for each transition and molecular species. Concerning the other sources, when the line emission profiles are clearly asymmetric, we are not able to reproduce those line profiles because:

- the modeling does not take the substructure into account (i.e. multiplicity of the massive dense cores, as for IRAS05358+3543), which is thus not reproduced correctly in the derived source model;

- outflows are not included in the model while they strongly influence the line profiles.
IRAS $18264-1152$
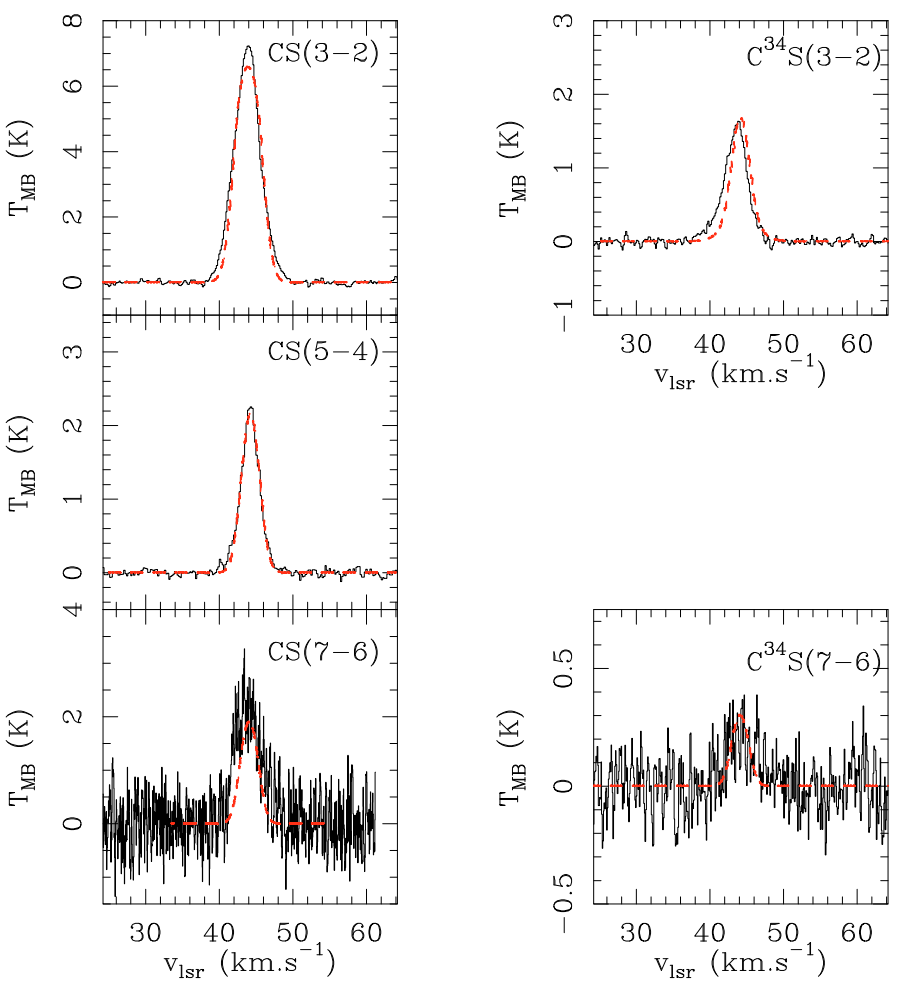

Fig. 8. Fit of the CS lines emission from IRAS18264-1152. The observed spectra are in black, fit in red.

Different turbulent $\left(v_{\mathrm{T}}\right)$ or infall $\left(v_{\text {infall }}\right)$ velocities are used to reproduce the different line emissions from each source. Some results for CS in the source IRAS18264-1152 are shown in Fig. 8.

\subsection{Uncertainties}

An overview of uncertainties in the modeling process that we used shows that precision on our abundance results is influenced by four main points: the signal-to-noise ratio, the total mass of the source in the model, its temperature, and the populations of energy levels of the molecule studied. Furthermore, an absolute error comes from the uncertainty on the dust opacity at millimeter wavelengths, but it disappears in the context of our work where the use of the same radiative transfer code and the same modeling process keep the comparison between each sources relevant. While signal-to-noise ratio is directly known from observations, the other uncertainties have to be explained and derived to determine how important they are.

At first, mass uncertainty comes from measurements of the flux at millimeter wavelengths. Considering calibration errors, we can assume that this value is known with $20 \%$ accuracy. Error on temperature is directly linked to this first error, in addition to the little variation in the bolometric luminosity induced. Nevertheless, results of the radiative transfer models show that extreme cases make the temperature vary by $3 \%$ at the maximum, and tend to compensate for the first error in the total mass. Even if temperature does not change a lot, its influence on energy level populations, combined with total mass (hence density) uncertainty, can be strong enough to be significant. These uncertainties are dependent on the molecule studied and the transition observed. As we noted previously, the errors can compensate for 
Table 5. Molecular abundances derived for all species within the 4 sources.

\begin{tabular}{|c|c|c|c|c|c|c|c|c|c|c|}
\hline Source & & & & W43MM1 & 18264 & 05358 & 18162 & $\mathrm{AFGL}_{2591^{a}}$ & $\mathrm{G} 31.41^{b}$ & $16293^{c}$ \\
\hline $\begin{array}{c}\text { Abundance } \\
\left(\times 10^{-10}\right)\end{array}$ & $\begin{array}{c}T \\
(\mathrm{~K})\end{array}$ & $\begin{array}{c}d^{d} \\
\left(10^{3} \mathrm{AU}\right)\end{array}$ & Line & & & & & & & \\
\hline \multirow{3}{*}{$\chi(\mathbf{C S})$} & 60 & 5.9 & $3-2$ & 36 & 4.0 & 2.3 & - & 100 & 70 & 5100 \\
\hline & 75 & 4.9 & $5-4$ & 30 & 0.26 & 1.3 & 1.0 & & & \\
\hline & 96 & 3.3 & $7-6$ & 160 & 1.1 & - & 4.6 & & & \\
\hline \multirow[t]{2}{*}{$\chi\left(\mathbf{C}^{34} \mathbf{S}\right)$} & 60 & 5.9 & $3-2$ & 1.8 & 0.32 & 0.27 & - & & & \\
\hline & 96 & 3.3 & $7-6$ & 8.0 & 0.50 & - & 0.27 & & & \\
\hline \multirow[t]{3}{*}{$\chi(\mathrm{OCS})$} & 72 & 5.2 & $8-7$ & 92 & 2.5 & 2.4 & 1.4 & 100 & 40 & 10000 \\
\hline & 76 & 4.8 & $13-12$ & 37 & 2.4 & 0.92 & 1.2 & & & \\
\hline & 96 & 3.3 & $19-18$ & 130 & 1.1 & 3.7 & 3.3 & & & \\
\hline$\chi\left(\mathbf{O C}^{34} \mathbf{S}\right)$ & 72 & 5.2 & $8-7$ & 4.6 & $<0.07$ & $<0.35$ & $<0.25$ & & & \\
\hline \multirow[t]{2}{*}{$\chi\left(\mathbf{H}_{2} \mathbf{S}\right)$} & 60 & 5.5 & $1_{10}-1_{01}$ & 3.0 & 1.3 & 1.2 & 3.1 & 80 & $\geq 30$ & 5300 \\
\hline & 96 & 3.3 & $2_{20}-2_{11}$ & 4.0 & 0.15 & 0.51 & 0.30 & & & \\
\hline$\chi\left(\mathbf{H}_{2}^{34} \mathbf{S}\right)$ & 60 & 5.5 & $1_{10}-1_{01}$ & $<0.19$ & 0.11 & $<0.13$ & 0.21 & & & \\
\hline \multirow[t]{4}{*}{$\chi(\mathbf{S O})$} & 51 & 8.7 & $3_{4}-2_{3}$ & 16 & 1.7 & 3.8 & 4.4 & 100 & 40 & 17000 \\
\hline & 62 & 6.4 & $5_{6}-4_{5}$ & 0.90 & 0.48 & 1.1 & 2.4 & & & \\
\hline & 75 & 4.8 & $65-5_{4}$ & 0.70 & 0.57 & 0.8 & 1.8 & & & \\
\hline & 96 & 3.3 & $8_{8}-7_{7}$ & 24 & 0.64 & - & 3.6 & & & \\
\hline \multirow[t]{2}{*}{$\chi\left({ }^{34} \mathbf{S O}\right)$} & 51 & 8.7 & $3_{4}-2_{3}$ & 0.80 & 0.11 & 0.33 & $<0.06$ & & & \\
\hline & 96 & 3.3 & $8_{8}-7_{7}$ & $<4.0$ & $<1.0$ & - & $<0.5$ & & & \\
\hline \multirow[t]{5}{*}{$\chi\left(\mathbf{S O}_{2}\right)$} & 57 & 7.2 & $5_{1,5}-4_{0,4}$ & 2.0 & 0.42 & 0.62 & 1.7 & 20 & 120 & 5400 \\
\hline & 71 & 5.3 & $10_{0,10}-9_{1,9}$ & 1.8 & 0.36 & 0.52 & 0.70 & & & \\
\hline & 76 & 4.8 & $11_{1,11}-10_{0,10}$ & - & 0.38 & - & 1.76 & & & \\
\hline & 112 & 2.7 & $14_{0,14}-13_{1,13}$ & - & 2.0 & 6.4 & 7.6 & & & \\
\hline & 112 & 2.7 & $18_{0,18}-17_{1,17}$ & 160 & $<6$ & - & 10.6 & & & \\
\hline$\chi\left({ }^{34} \mathbf{S O}_{2}\right)$ & 57 & 7.2 & $5_{1,5}-4_{0,4}$ & $<0.33$ & $<0.44$ & $<16$ & $<0.22$ & & & \\
\hline
\end{tabular}

${ }^{a}$ Mean abundances from van der Tak et al. (2003); ${ }^{b}$ mean abundances from Hatchell et al. (1998); ${ }^{c}$ mean abundances from Wakelam et al. (2004)a; ${ }^{d}$ indicative corresponding distance to the center for the source IRAS18162-2048.

one another, especially when variation in the level energy populations of a transition are going the same way.

Finally, we derived rough values of uncertainties by testing the temperature and energy-level population variations induced by flux errors at millimeter range. Results show that our modeling process has a relative precision between $25 \%$ and $30 \%$, depending on the considered molecular line emission. This range of values does not take the signal-to-noise ratio of the observations into account. Concerning $\mathrm{SO}_{2}$ transitions, populations of the energy levels vary widely, making the uncertainty increase up to $40 \%$, even $70 \%$, for the $5_{1,5}-4_{0,4}$ where populations show an opposite behavior against temperature variations in our models. Furthermore, we note that optically thin emission was assumed, whereas some of them are clearly thick (see Table 10). That is why abundances derived in this case must be treated as indicating values for the order of magnitude.

\subsection{Molecular abundances: results}

The derived abundances for each molecule and each source are given in Table 5 for different layers. Each layer is characterized by a temperature (and by a density, but the population of highenergy levels is sensitive to the temperature for dense gas tracers). We also give the strongest line emission in that layer (but of course lines are excited over several layers). Results are plotted in Fig. 9 versus the energy of the upper level of the transition. Obviously emission lines coming from warmer regions (more than $120 \mathrm{~K}$ ), e.g. involving higher upper energy levels, are missing for probing the most inner regions.

The $\mathrm{H}_{2} \mathrm{~S}$ abundance is roughly the same in the 4 objects studied $\left(1.6-3.610^{-10}\right)$ in the outer region $(T \sim 60 \mathrm{~K})$, showing more variation $\left(0.16-410^{-10}\right)$ in the warmer inner parts
$(T \sim 100 \mathrm{~K})$ : in IRAS18264-1152, IRAS05358+3543, and IRAS18162-2048, the abundance is one order of magnitude lower while it remains constant in W43MM1.

An obvious trend is seen for $\mathrm{SO}_{2}$, likely because the energy ladder of this molecule is sampled best. Its abundance is roughly constant (or decreases for W43MM1) in colder layers $\left(T<80 \mathrm{~K}\right.$, e.g. $>5 \times 10^{3}$ AU for the source IRAS18162-2048, and $E_{\text {up }}<35 \mathrm{~K}$ ), then increases by one order of magnitude in warmer layers (hence outer to more inner parts), e.g. $\sim 3 \times 10^{3} \mathrm{AU}$ for the same source. For all the other molecules, as fewer transitions have been observed, i.e. the energy ladder is not completely covered, so no obvious trends are seen. In spite of an insufficient energy coverage, the derived OCS abundance might weakly follow the same trend as seen for CS, except in the source IRAS18264-1152. We note that the sources IRAS18264-1152 and IRAS05358+3543 exhibit the same abundance variations for $\mathrm{SO}$ and $\mathrm{SO}_{2}$.

In the three less evolved objects, CS and OCS are the most abundant species in the colder outer region, while it is SO for IRAS18162-2048 (for that source OCS is less abundant than SO and no CS 3-2 has been observed), $\mathrm{SO}_{2}$ being the least abundant.

Reproducing the whole CS and $\mathrm{C}^{34} \mathrm{~S}$ emission is impossible with the same isotopic ratio for all the sources. The derived isotopic ratio $\left[{ }^{32} \mathrm{~S} /{ }^{34} \mathrm{~S}\right]$ is $9-12,10-15,15-20$, and 20, respectively, for IRAS05358+3543, IRAS18264-1152, IRAS18162-2048, and W43MM1. The Solar System abundance ratio is 22.5 (Kahane et al. 1988), but Chin et al. (1996) found ratios increasing from 14 to 35 with the distance to the Galactic center, though this contradicts Nilsson et al. (2000) who derived a ratio of 11 towards the very distant source W49N. 

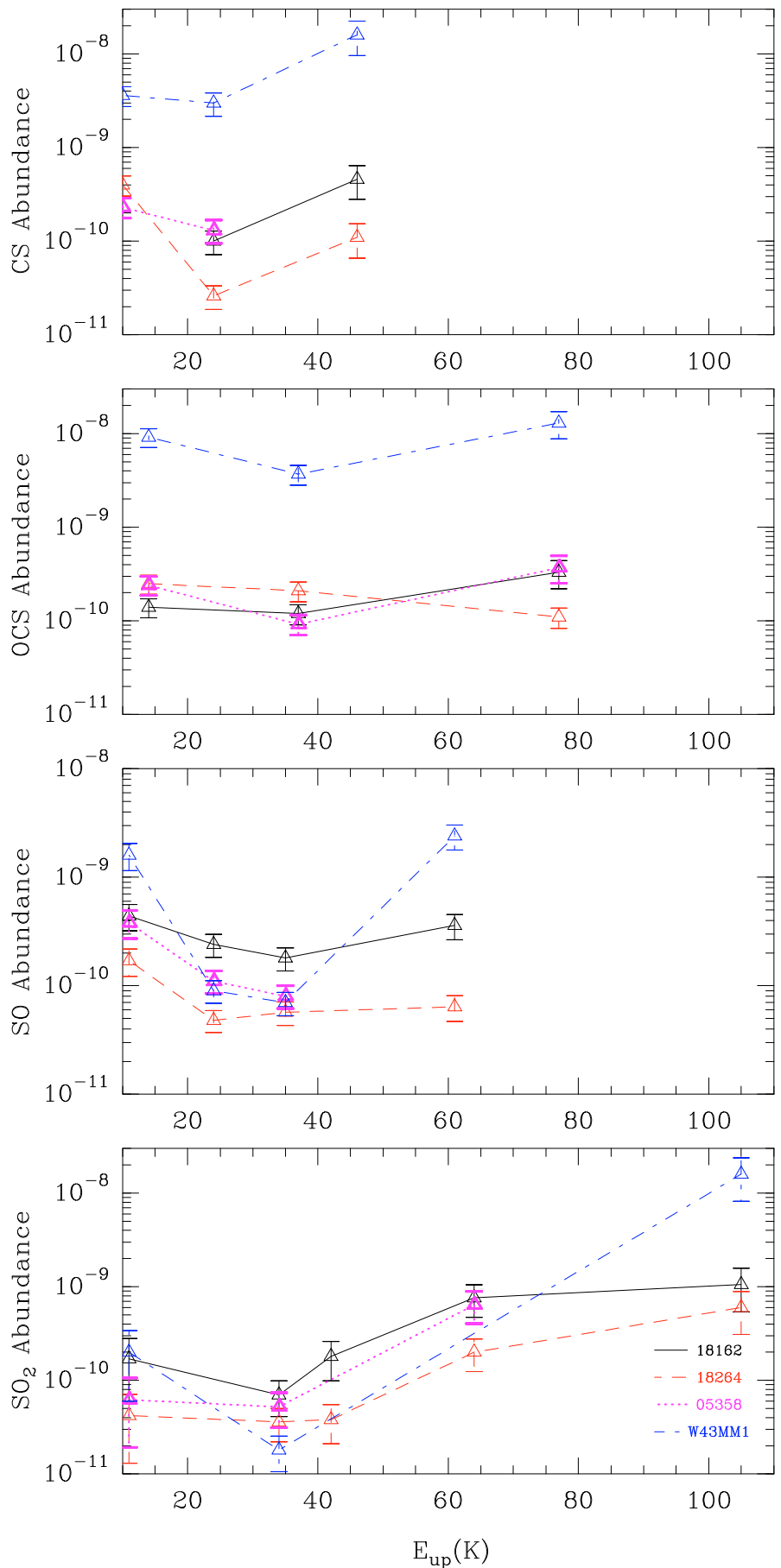

Fig. 9. Abundances of $\mathrm{CS}$, OCS, $\mathrm{SO}$, and $\mathrm{SO}_{2}$ (relative to $\mathrm{H}_{2}$ ) versus the energy of the upper level of the transition given in Table 5 for the 4 sources.

\section{Discussion}

\subsection{W43MM1: a special case}

W43MM1 is clearly different from the other sources, with the highest abundances (relative to $\mathrm{H}_{2}$ ) of CS, OCS, $\mathrm{SO}$ (except in the flattening region); the $\mathrm{SO}_{2}$ abundance in W43MM1 is the highest in the warmer inner part. Moreover the plots (see Figs. 10 and 11) of the CS and OCS molecular ratios relative to $\mathrm{H}_{2} \mathrm{~S}$ underline this difference, showing high ratios for that source compared to the others.
The reason for this might be that W43MM1, though a very young source, has already developed a hot core with temperatures higher than $200 \mathrm{~K}$ (e.g. $\mathrm{CH}_{3} \mathrm{CN}$ lines observed by Motte et al. 2003). This hot core region might also be at the origin of the unidentified lines described in Sect. 4.1. The presence of this hot core might also explain the high abundance of $\mathrm{H}_{2} \mathrm{~S}$ and OCS molecules whose evaporation from grain surface could be important (Wakelam et al. 2004a), leading to the high abundance of SO. The CS case is different because this molecule is already present in the gas before the evaporation. Actually, the CS chemistry is closely linked to the atomic carbon abundance, which might be higher in W43MM1 than in the other sources.

The very high $\mathrm{SO}_{2}$ abundance close to the center of W43MM1 may come from to strong shocks occurring in the inner part of the envelope. That W43MM1 is a very young source (as indicated by the possibly detected infall, see Sect. 5.2) suggests that this massive dense core harbors one or several highmass protostars with powerful outflows, which might induce shocked regions. Strong shocks can convert $\mathrm{SO}$ into $\mathrm{SO}_{2}$ (see Hatchell et al. 1998), then leading to a ratio of unity, as observed in the other sources where the process has already taken place, transforming the ratio across the envelope. As a result, shock chemistry might contribute to the $\mathrm{SO}_{2}$ formation process.

Actually, IRAS05358+3543 harbors a hot core, too (Leurini et al. 2007), but very likely one more compact than the one of W43MM1 (Motte et al. 2003), hence more diluted in the beam of our observations. Moreover, Leurini et al. (2007) suggests that the IRAS05358+3543 hot core might not produce a very rich chemistry. Indeed, these authors underlined differences between its molecular spectrum and the one of typical massive hot cores.

\subsection{Molecular ratios as a diagnostic tool of evolution?}

Sulfur is released into the gas phase in the form of $\mathrm{H}_{2} \mathrm{~S}$, which is subsequently transformed into $\mathrm{SO}$ and later into $\mathrm{SO}_{2}$ via neutralneutral reaction at $100 \mathrm{~K}$ on time scales of $\sim 10^{3} \mathrm{yr}$ (Wakelam et al. 2004a). The initial destruction of $\mathrm{H}_{2} \mathrm{~S}$ by $\mathrm{H}_{3} \mathrm{O}^{+}$is even more efficient in the high-mass objects than in low-mass protostars, as water is more abundant there (see van der Tak et al. 2006). At higher temperature $(T \geq 300 \mathrm{~K}), \mathrm{H}_{2} \mathrm{~S}$ can be produced again. Once in the gas phase, OCS is destroyed later than $\mathrm{H}_{2} \mathrm{~S}$ (by $\mathrm{S}^{+}$and cosmic rays). CS destruction at low temperature produces $\mathrm{SO}$ and $\mathrm{SO}_{2}$ too, while $\mathrm{CS}$ is produced again at a higher temperature from OCS. The time dependence of these process might allow us to see an evolution in the abundance along the protostar evolutionary track.

Actually no evolution is obviously seen for the abundances relative to $\mathrm{H}_{2}$, even if they differ significantly from source to source. Nevertheless, W43MM1 shows larger abundances of CS, OCS, and SO compared to IRAS18264-1152 and IRAS05358+3543 (see Sect. 6.4).

We investigated possible correlations of the molecular abundance ratios with the order of evolution of our 4 objects for two different layers: one around $T=60-70 \mathrm{~K}$ and a more inner one around $T=100 \mathrm{~K}$. To be coherent, we only consider values derived for the same layer, i.e same temperature (e.g. transitions CS 5-4 and OCS 13-12 with $\mathrm{E}_{\mathrm{up}}=24-37 \mathrm{~K}$, hence mostly excited in the $75 \mathrm{~K}$ layer). Figures $10-13$ show that some global trend appears, either a decrease or an increase with the evolution, but depending on the layer.

We first compared the molecular abundances relative to $\mathrm{H}_{2} \mathrm{~S}$ whose abundance is assumed to remain globally constant during the studied evolutionary period and hence comparable from source to source. In the colder region $(T=60 \mathrm{~K}$, Fig. 10), 


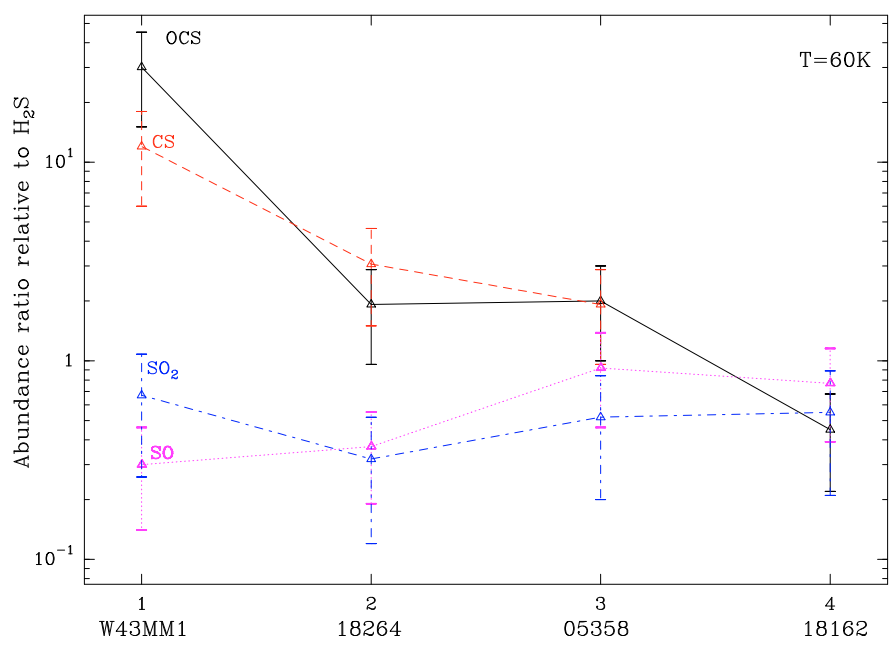

Fig. 10. Abundance ratio of OCS, CS, $\mathrm{SO}$, and $\mathrm{SO}_{2}$ relative to $\mathrm{H}_{2} \mathrm{~S}$ for each source in the layer at $T=60 \mathrm{~K}$. The sources are ordered according to the SED classification.

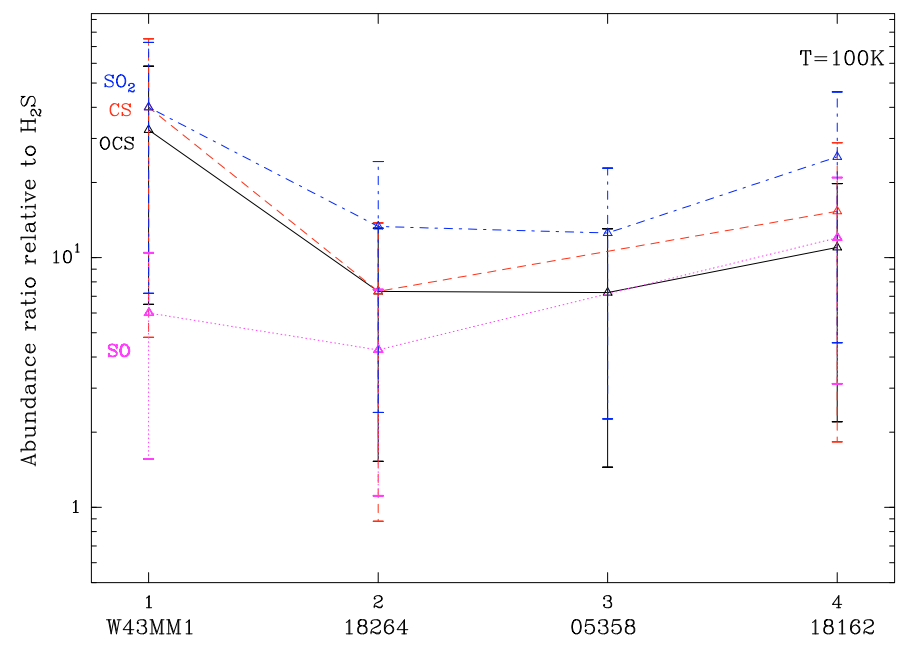

Fig. 11. Abundance ratio of OCS, CS, $\mathrm{SO}$, and $\mathrm{SO}_{2}$ relative to $\mathrm{H}_{2} \mathrm{~S}$ for each source in the layer at $T=100 \mathrm{~K}$. The sources are ordered according to the SED classification.

the $\left[\mathrm{OCS} / \mathrm{H}_{2} \mathrm{~S}\right]$ ratio is clearly decreasing from W43MM1 to IRAS18162-2048 by a factor 10 . This trend is also visible for $\mathrm{CS}$. The $\left[\mathrm{SO} / \mathrm{H}_{2} \mathrm{~S}\right]$ and $\left[\mathrm{SO}_{2} / \mathrm{H}_{2} \mathrm{~S}\right]$ ratios remain roughly constant; a small increase might actually be present for SO. In the inner, hence warmer, part of the envelope ( $T=100 \mathrm{~K}$, Fig. 11), drawing conclusions is more difficult, because not all 4 sources were observed in the same transitions. Moreover, W43MM1 stands out as it shows the highest ratio (except for $\mathrm{SO}$ ), independent of the trend seen for the three other objects. Actually, according to the temperature and density distributions derived by our model (see Fig. 7), the $100 \mathrm{~K}$ layer for W43MM1 is closer to the center of the massive core than in the other sources, so it corresponds to a higher density. But the other reason could be that the hot core is dominating. Therefore, considering the three other sources, an increase in the different ratios relative to $\mathrm{H}_{2} \mathrm{~S}$ from IRAS181264 to IRAS18162-2048 is seen. Comparing the 60 and $100 \mathrm{~K}$ regions, the main difference is for OCS and CS: ratios decrease at $T=60 \mathrm{~K}$, but increase at higher temperature.

Compared to OCS (Fig. 12), the abundance of CS does not show any trend, neither at $76 \mathrm{~K}$ nor at $96 \mathrm{~K}$. At $70-75 \mathrm{~K}$, the $[\mathrm{SO} / \mathrm{OCS}]$ and $\left[\mathrm{SO}_{2} / \mathrm{OCS}\right]$ ratios increase by at least one order of magnitude along the derived evolutionary track. At higher temperature, hence in the inner regions, the same trend is observed for the $[\mathrm{SO} / \mathrm{OCS}]$ ratio, while $\left[\mathrm{SO}_{2} / \mathrm{OCS}\right]$ remains approximately constant. For both probed layers, the $[\mathrm{CS} / \mathrm{SO}]$ and $\left[\mathrm{SO}_{2} / \mathrm{SO}\right]$ ratios decrease (Fig. 13), the variation being more important at $60 \mathrm{~K}$.

Cross-comparison of the plots for outer regions reveals that the OCS (and maybe CS) abundance (relative to $\mathrm{H}_{2} \mathrm{~S}$ ) decreases, while the $\mathrm{SO}$ and $\mathrm{SO}_{2}$ relative abundance (compared to other molecules) increase along the object evolution. This trend is less obvious at higher temperature, maybe because in these regions the $\mathrm{H}_{2} \mathrm{~S}$ transformation process into $\mathrm{SO}$ and $\mathrm{SO}_{2}$ (Wakelam et al. 2004a,b) is becoming less efficient, as it gets close to the completion: the new $\mathrm{SO}$ and $\mathrm{SO}_{2}$ molecules have already been produced in the inner parts. This hypothesis is strengthened by the larger $\mathrm{SO}_{2}$ abundance measured in all the objects for the higher temperatures, i.e. in warmer environments. Nevertheless, any conclusions for the inner parts are tricky, because of the incomplete energy ladder coverage of our observations and so the lack of very high-energy transitions.

\subsection{Comparison with previous studies}

A comparison between abundances derived for low- and highmass protostars is not straightforward, because of the different composition of the ices (from which $\mathrm{H}_{2} \mathrm{~S}$ evaporates) and of the different physical conditions (density and temperature; e.g. density can be two orders of magnitude higher for the same temperature) as stressed by Wakelam et al. (2004a). Nevertheless, Table 5 also gives abundances for AFGL2591 (van der Tak et al. 2003), a mid-IR bright HMPO ( $L_{\text {bol }} 2-10$ times larger, warmer SED with higher $F_{12}$ and $F_{35} / F_{\text {total }}$, hence more evolved object, and G31.41+0.31 (Hatchell et al. 1998), a massive hot core and IRAS16293 (Wakelam et al. 2004a). We stress that those values are mean abundances across the whole envelope. Values for AFGL2591 and G31.41 differ a lot for $\mathrm{H}_{2} \mathrm{~S}$ and $\mathrm{SO}_{2}$ (by at least a factor 10) but are comparable for CS, OCS, and SO to the abundances found in W43MM1. The other sources from our sample exhibit abundances that are one or two order of magnitude lower. Abundances in low-mass protostar are 100-1000 times larger. Clearly the same set of lines has to be observed towards these sources and the same modeling applied to be able to be compared with our sample.

The sulfur chemistry was studied by van der Tak et al. (2003) in the envelopes of more evolved massive protostellar objects, mid-IR bright HMPOs. The abundances they derived are 10100 times higher than ours (see Table 5). They concluded that shock chemistry is unlikely to contribute and that OCS is a major sulfur carrier in the ices. The molecular ratios computed from their work do not show significant variations from source to source.

From our work, the total abundance of sulfur $\left(\mathrm{CS}+\mathrm{SO}+\mathrm{SO}_{2}+\mathrm{H}_{2} \mathrm{~S}+\mathrm{OCS}\right)$ can be estimated as $210^{-9}-510^{-8}$, several orders of magnitude lower than the corresponding solar abundance $\left(3.4 \times 10^{-5}\right)$ and the value derived in the low-mass hot core IRAS16293 by Wakelam et al. $(2004 a)\left(2.8 \times 10^{-6}\right)$. It is likely that we do not probe the very inner parts of the protostars where the S-bearing molecules are fully evaporated from the grain mantles. Hypotheses to explain such depletion of sulfur (including the one previously mentioned) will be explored in another paper on the chemistry. 

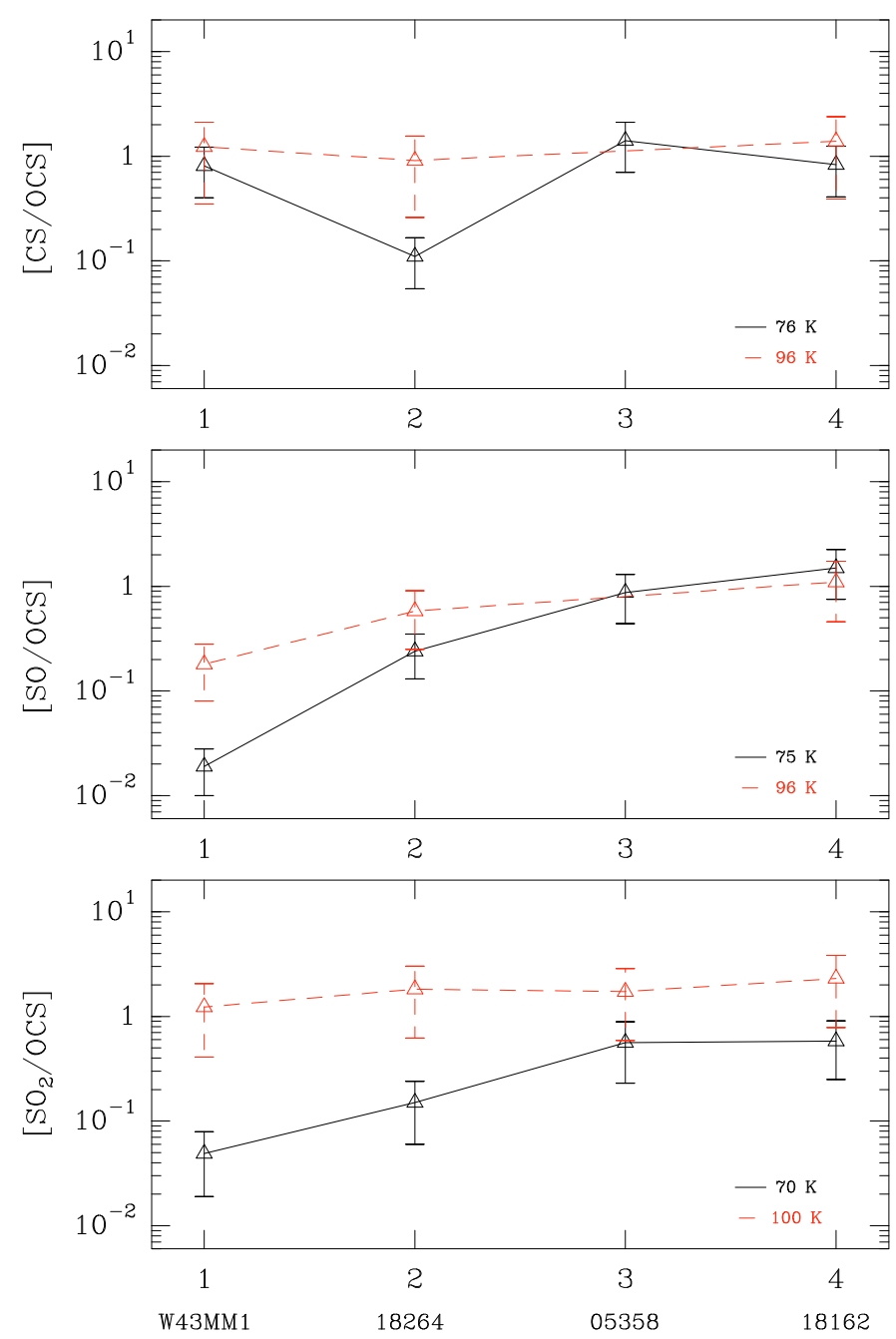

Fig. 12. Abundance ratio of $\mathrm{CS}, \mathrm{SO}$, and $\mathrm{SO}_{2}$ relative to $\mathrm{OCS}$ for each source in two different layers. The sources are ordered according to the SED classification.

\section{Conclusion}

We have presented here new observations of sulfur-bearing species toward 4 massive dense cores. Different gas components, revealing outflows or multiplicity, are detected in IRAS05358+3543, IRAS18264-1152, and IRAS18162-2048. A strong infall of more than $2 \mathrm{~km} \mathrm{~s}^{-1}$ towards W43MM1 might be observed, leading to an impressive kinematic mass-infall rate. The W43MM1 study shows that a hot core may appear earlier than expected in the evolution.

An evolutionary classification derived from the calculated SEDs is proposed from W43MM1 to IRAS18162-2048, and is likely to be observed in the molecular abundances, too. More precisely, molecular ratios like $\left[\mathrm{OCS} / \mathrm{H}_{2} \mathrm{~S}\right],\left[\mathrm{CS} / \mathrm{H}_{2} \mathrm{~S}\right]$, [SO/OCS], $\left[\mathrm{SO}_{2} / \mathrm{OCS}\right],[\mathrm{CS} / \mathrm{SO}]$, and $\left[\mathrm{SO}_{2} / \mathrm{SO}\right]$, using lowenergy transitions, might be good indicators of evolution depending on the layers probed by the observed molecular transitions: obvious trends along the massive core evolution are seen for colder outer regions at $60 \mathrm{~K}$, but drawing definite conclusions is more difficult for the inner regions. Observations of molecular emission from warmer layers, implying higher upper energy levels must be included. A follow-up with APEX telescope should be made to have access to these higher frequencies. Of course, the source multiplicity due to the distance is problematic, so only ALMA will provide a definitive answer.
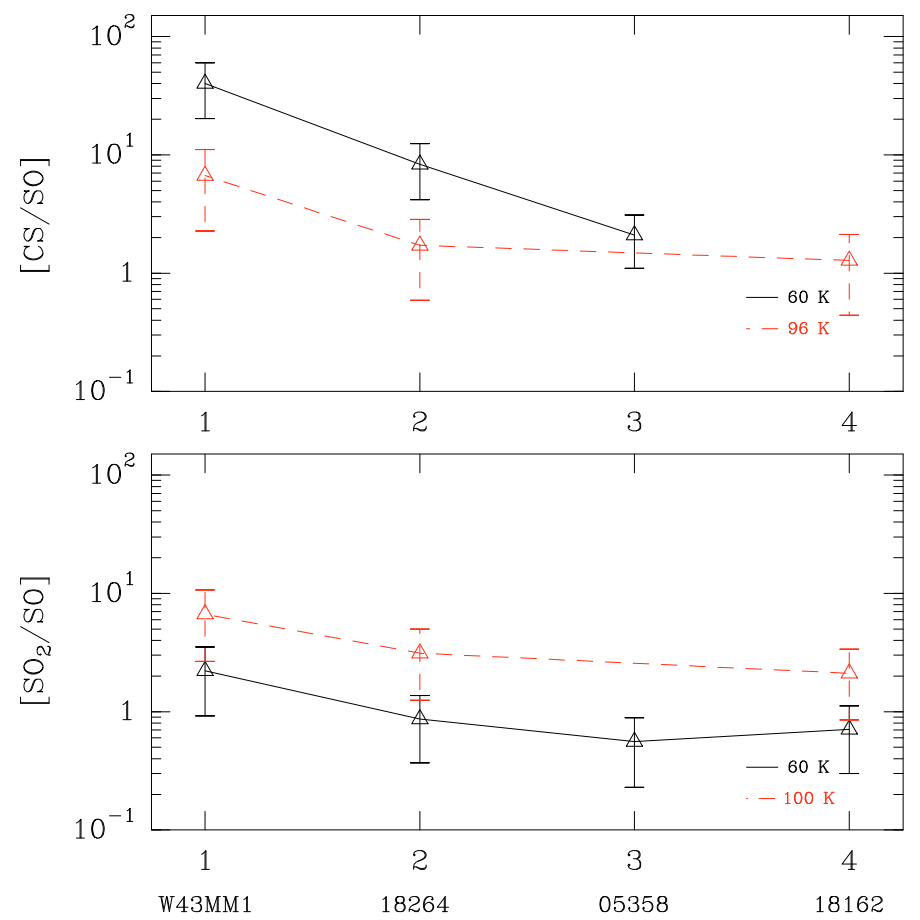

Fig. 13. Abundance ratio of $\mathrm{CS}$ and $\mathrm{SO}_{2}$ relative to $\mathrm{SO}$ for each source in two different layers. The sources are ordered according to the SED classification.

Nevertheless, specific chemical modeling will be done for these sources in a forthcoming paper (Wakelam et al., in preparation) to explain the observed molecular ratios and to try to date the studied objects.

\section{References}

Andre, P., Ward-Thompson, D., \& Barsony, M. 2000, Protostars and Planets IV, 59

Benedettini, M., Molinari, S., Testi, L., et al. 2004, MNRAS, 347, 295

Beuther, H., \& Schilke, P. 2004, Science, 303, 1167

Beuther, H., Schilke, P., Gueth, F., et al. 2002a, A\&A, 387, 931

Beuther, H., Schilke, P., Menten, K. M., et al. 2002b, ApJ, 566, 945

Beuther, H., Schilke, P., Sridharan, T. K., et al. 2002c, A\&A, 383, 892

Beuther, H., Churchwell, E. B., McKee, C. F., et al. 2007a, in Protostars and Planets V, ed. B. Reipurth, D. Jewitt, \& K. Keil, 165

Beuther, H., Leurini, S., Schilke, P., et al. 2007b, A\&A, 466, 1065

Bonnell, I. A., \& Bate, M. R. 2006, MNRAS, 370, 488

Charnley, S. B. 1997, ApJ, 481, 396

Chin, Y.-N., Henkel, C., Whiteoak, J. B., Langer, N., \& Churchwell, E. B. 1996, A\&A, 305, 960

Doty, S. D., van Dishoeck, E. F., van der Tak, F. F. S., et al. 2002, A\&A, 389, 446

Fuller, G. A., Williams, S. J., \& Sridharan, T. K. 2005, A\&A, 442, 949

Guilloteau, S., \& Lucas, R. 2000, in Imaging at Radio through Submillimeter

Wavelengths, ed. J. G. Mangum, \& S. J. E. Radford, ASP Conf. Ser., 217, 299

Hatchell, J., Thompson, M. A., Millar, T. J., et al. 1998, A\&A, 338, 713

Hogerheijde, M. R., \& van der Tak, F. F. S. 2000, A\&A, 362, 697

Hunter, T. R., Churchwell, E., Watson, C., et al. 2000, AJ, 119, 2711

Jenness, T., Scott, P. F., \& Padman, R. 1995, MNRAS, 276, 1024

Kahane, C., Gomez-Gonzalez, J., Cernicharo, J., et al. 1988, A\&A, 190, 167

Krumholz, M. R., \& McKee, C. F. 2008, Nature, 451, 1082

Kurtz, S., Churchwell, E., \& Wood, D. O. S. 1994, ApJS, 91, 659

Leurini, S., Beuther, H., Schilke, P., et al. 2007, A\&A, 475, 925

Lis, D. C., Keene, J., Phillips, T. G., et al. 2001, ApJ, 561, 823

Longmore, S. N., Burton, M. G., Minier, V., et al. 2006, MNRAS, 369, 1196

Mardones, D., Myers, P. C., Tafalla, M., et al. 1997, ApJ, 489, 719

Marseille, M., Bontemps, S., Herpin, F., van der Tak, F. F. S., \& Purcell, C. R. 2008, A\&A, 488, 579 
Menten, K. M., Pillai, T., \& Wyrowski, F. 2005, in Massive Star Birth: A Crossroads of Astrophysics, ed. R. Cesaroni, M. Felli, E. Churchwell, \& M. Walmsley, IAU Symp., 227, 23

Millar, T. J., MacDonald, G. H., \& Gibb, A. G. 1997, A\&A, 325, 1163

Minier, V., Burton, M. G., Hill, T., et al. 2005, A\&A, 429, 945

Motte, F., \& André, P. 2001, A\&A, 365, 440

Motte, F., \& Hennebelle, P. 2009, in EAS Publ. Ser. 34, ed. L. Pagani, \& M. Gerin, 195

Motte, F., Schilke, P., \& Lis, D. C. 2003, ApJ, 582, 277

Motte, F., Bontemps, S., Schilke, P., et al. 2005, in Massive Star Birth: A Crossroads of Astrophysics, ed. R. Cesaroni, M. Felli, E. Churchwell, \& M. Walmsley, IAU Symp., 227, 151

Motte, F., Bontemps, S., Schilke, P., et al. 2007, A\&A, 476, 1243

Myers, P. C., Mardones, D., Tafalla, M., Williams, J. P., \& Wilner, D. J. 1996, ApJ, 465, L133

Nilsson, A., Hjalmarson, Å., Bergman, P., et al. 2000, A\&A, 358, 257

Qiu, K., Zhang, Q., Beuther, H., et al. 2007, ApJ, 654, 361

Shepherd, D. S., Testi, L., \& Stark, D. P. 2003, ApJ, 584, 882
Sridharan, T. K., Beuther, H., Schilke, P., Menten, K. M., \& Wyrowski, F. 2002, ApJ, 566, 931

Stecklum, B., Feldt, M., Richichi, A., Calamai, G., \& Lagage, P. O. 1997, ApJ, 479,339

Su, Y.-N., Zhang, Q., \& Lim, J. 2004, ApJ, 604, 258

Tan, J. C., \& McKee, C. F. 2002, in Hot Star Workshop III: The Earliest Phases of Massive Star Birth, ed. P. Crowther, ASP Conf. Ser., 267, 267

van der Tak, F. F. S., Boonman, A. M. S., Braakman, R., et al. 2003, A\&A, 412, 133

van der Tak, F. F. S., Walmsley, C. M., Herpin, F., et al. 2006, A\&A, 447, 1011

Vastel, C., Polehampton, E. T., Baluteau, J.-P., et al. 2002, ApJ, 581, 315

Wakelam, V., Caselli, P., Ceccarelli, C., Herbst, E., \& Castets, A. 2004a, A\&A, 422, 159

Wakelam, V., Castets, A., Ceccarelli, C., et al. 2004b, A\&A, 413, 609

Williams, J. P., Blitz, L., \& McKee, C. F. 2000, Protostars and Planets IV, 97

Wolf, S., Henning, T., \& Stecklum, B. 1999, A\&A, 349, 839

Yamashita, T., Suzuki, H., Kaifu, N., et al. 1989, ApJ, 347, 894 
F. Herpin et al.: S-bearing molecules in massive dense cores, Online Material $p 1$

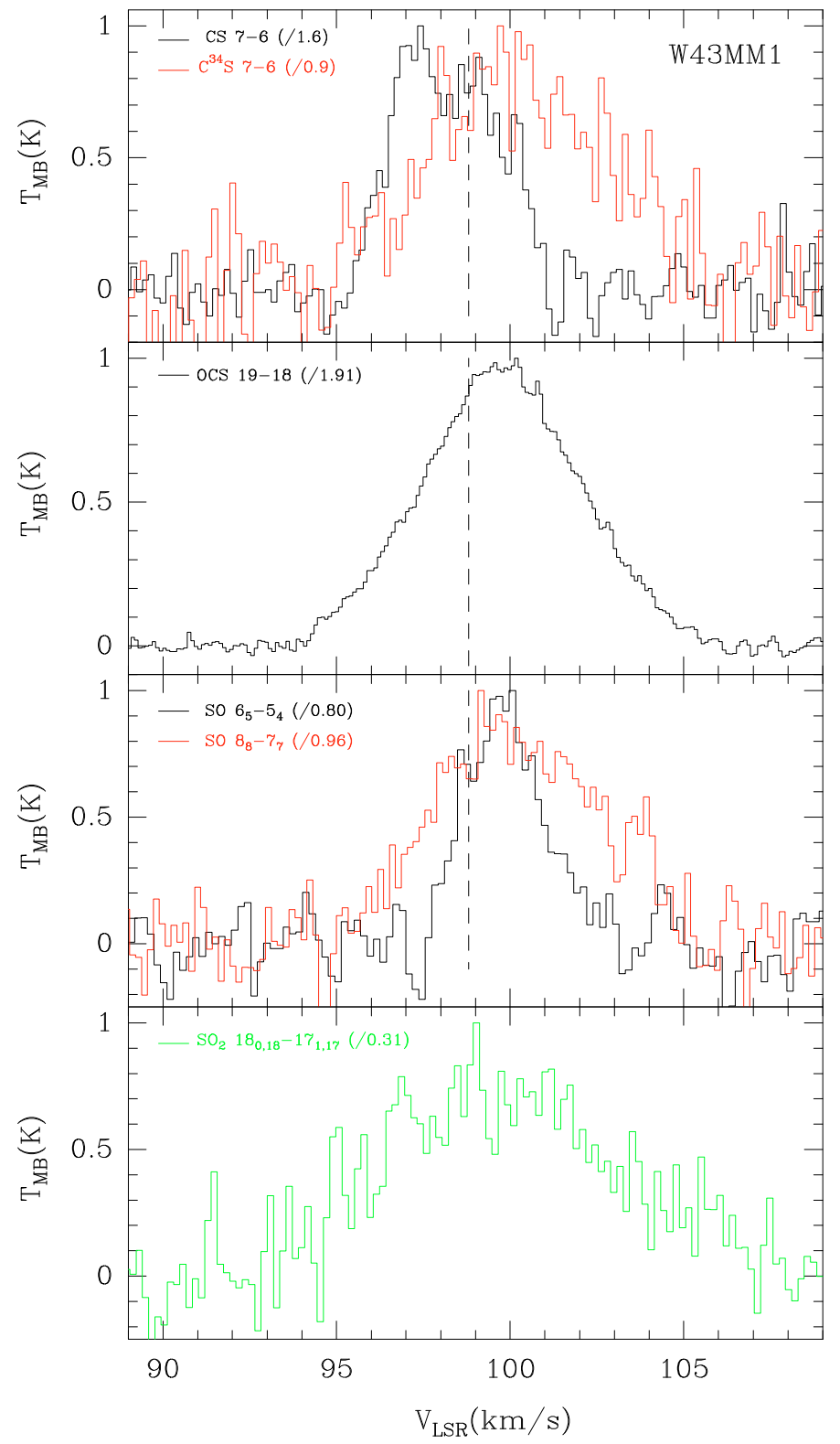

Fig. 14. Normalized emission of $\mathrm{CS}, \mathrm{H}_{2} \mathrm{~S}$, OCS, $\mathrm{SO}, \mathrm{SO}_{2}$ (and isotopic species) lines from W43MM1. Spectra velocity resolutions are 0.10$0.19 \mathrm{~km} \mathrm{~s}^{-1}$. The dashed line shows the source LSR velocity. 
F. Herpin et al.: S-bearing molecules in massive dense cores, Online Material $p 2$

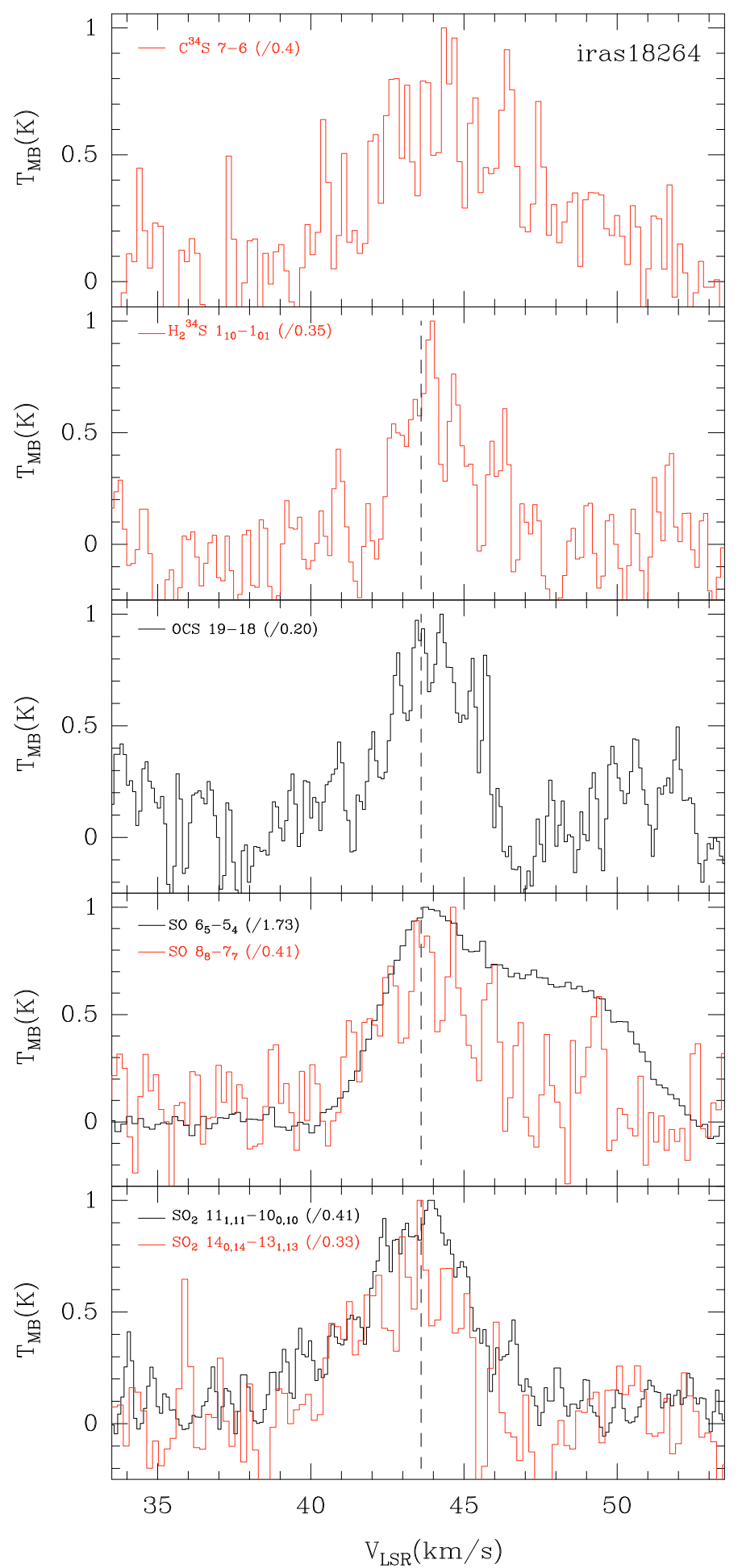

Fig. 15. Normalized emission of $\mathrm{CS}, \mathrm{H}_{2} \mathrm{~S}$, OCS, SO, $\mathrm{SO}_{2}$ (and isotopic species) lines from IRAS18264-1152. Spectra velocity resolutions are $0.10-0.19 \mathrm{~km} \mathrm{~s}^{-1}$. The dashed line shows the source LSR velocity. 
F. Herpin et al.: S-bearing molecules in massive dense cores, Online Material p 3

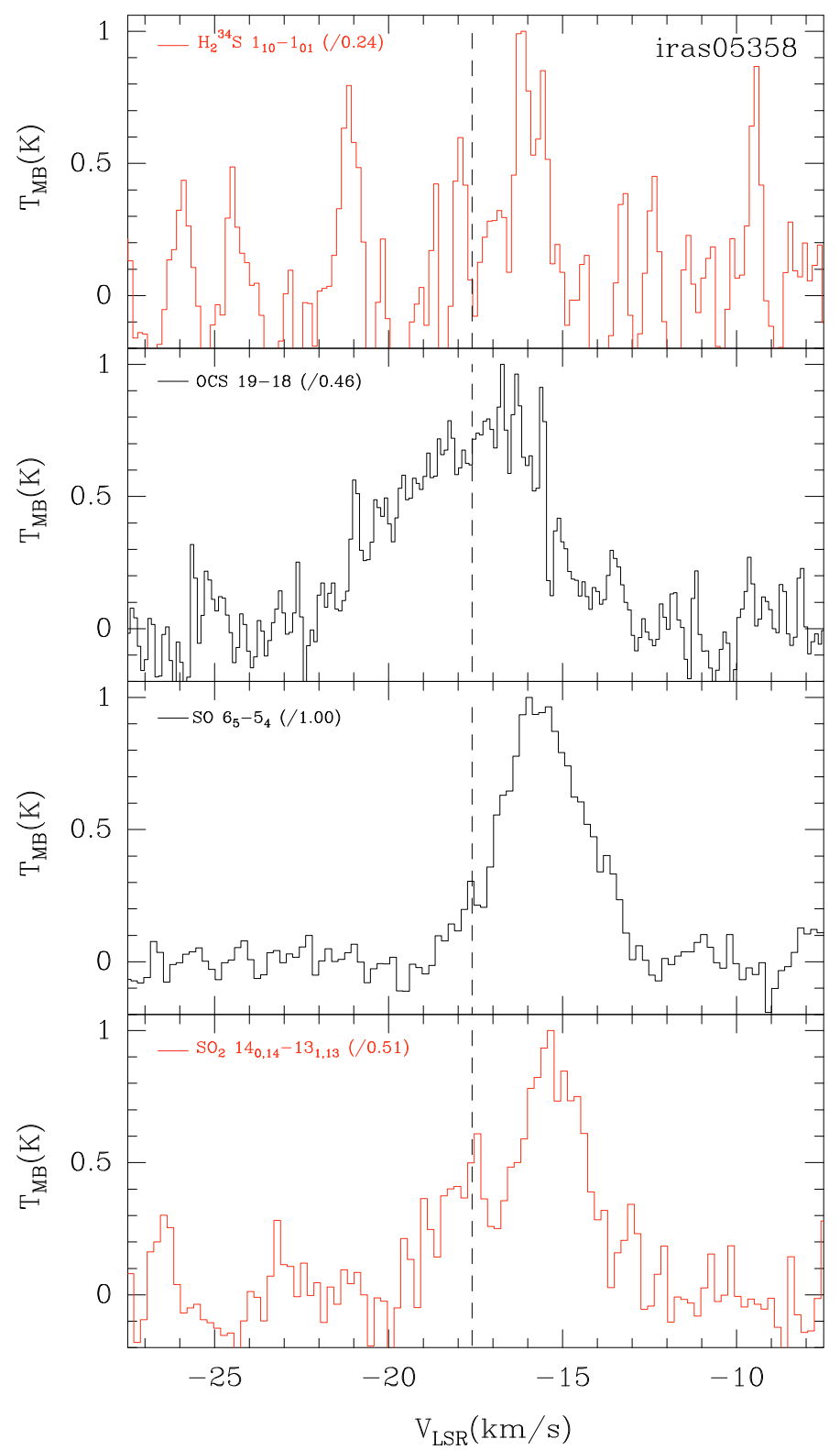

Fig. 16. Normalized emission of $\mathrm{CS}, \mathrm{H}_{2} \mathrm{~S}, \mathrm{OCS}, \mathrm{SO}, \mathrm{SO}_{2}$ (and isotopic species) lines from IRAS05358+3543. Spectra velocity resolutions are $0.10-0.19 \mathrm{~km} \mathrm{~s}^{-1}$. The dashed line shows the source LSR velocity. 
F. Herpin et al.: S-bearing molecules in massive dense cores, Online Material p 4

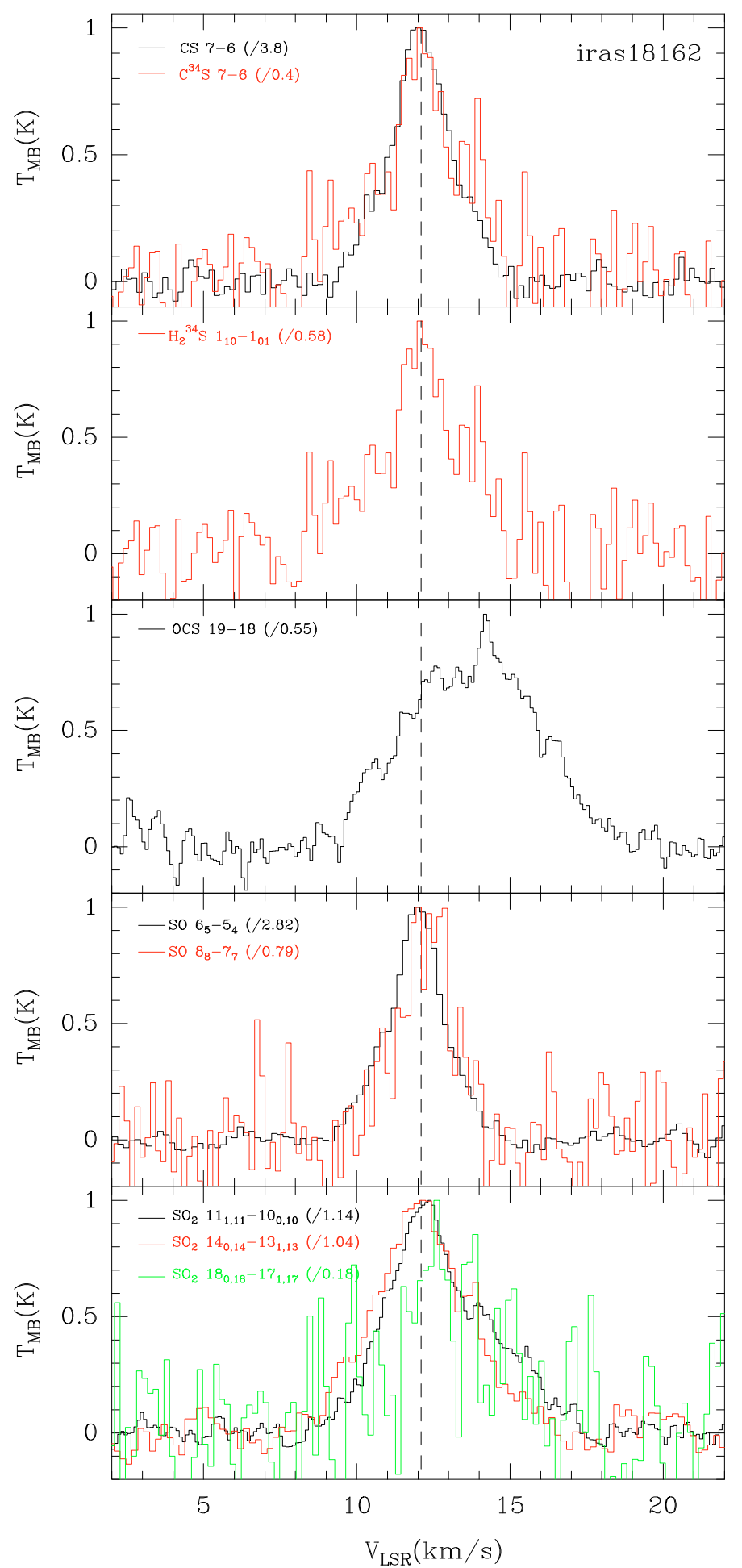

Fig. 17. Normalized emission of $\mathrm{CS}, \mathrm{H}_{2} \mathrm{~S}$, OCS, SO, $\mathrm{SO}_{2}$ (and isotopic species) lines from IRAS18162-2048. Spectra velocity resolutions are $0.10-0.19 \mathrm{~km} \mathrm{~s}^{-1}$. The dashed line shows the source LSR velocity. 
F. Herpin et al.: S-bearing molecules in massive dense cores, Online Material p 5

Table 6. Observed line emissions parameters for W43MM1.

\begin{tabular}{|c|c|c|c|c|c|c|c|c|}
\hline Species & Transition & $\begin{array}{c}\Delta v \\
\mathrm{~km} \mathrm{~s}^{-1}\end{array}$ & $\begin{array}{c}T_{\mathrm{mb}} \\
\mathrm{K}\end{array}$ & $\begin{array}{c}\sigma \\
\mathrm{mK}\end{array}$ & $\int_{\mathrm{K} \cdot \mathrm{km} \mathrm{s}^{-1}} T_{\mathrm{mb}} \delta v$ & $\begin{array}{c}\Delta v_{3 \sigma} \\
\mathrm{km} \mathrm{s}^{-1}\end{array}$ & $\begin{array}{c}\Delta v_{1 / 2} \\
\mathrm{~km} \mathrm{~s}^{-1}\end{array}$ & Comment \\
\hline \multirow[t]{3}{*}{$\mathrm{CS}$} & $3-2$ & $9.3(0.2)$ & 4.01 & 36 & $22.90(0.15)$ & 15.69 & 9.21 & $\bar{a}$ \\
\hline & $5-4$ & $9.68(0.04)$ & 6.85 & 62 & $61.6(0.3)$ & 14.50 & 9.36 & $a$ \\
\hline & $7-6$ & $4.2(0.3)$ & 1.2 & 255 & $5.4(0.7)$ & 4.5 & 4.5 & $b$ \\
\hline \multirow{2}{*}{$\mathrm{C}^{34} \mathrm{~S}$} & $3-2$ & $5.69(0.02)$ & 2.12 & 42 & $12.96(0.06)$ & 11.23 & 6.02 & \\
\hline & $7-6$ & $6.1(0.3)$ & 1.05 & 269 & $4.8(0.2)$ & 2.7 & 5.6 & \\
\hline \multirow[t]{4}{*}{ SO } & $3_{4}-2_{3}$ & $6.10(0.01)$ & 2.66 & 23 & $17.52(0.03)$ & 13.24 & 6.62 & \\
\hline & $5_{6}-4_{5}$ & $4.40(0.06)$ & 1.14 & 65 & $5.10(0.07)$ & 7.22 & 4.51 & \\
\hline & $6_{5}-5_{4}$ & $2.6(0.1)$ & 0.8 & 85 & $2.01(0.09)$ & 3.47 & 2.54 & \\
\hline & $8_{8}-7_{7}$ & $5.4(0.3)$ & 0.6 & 184 & $2.8(0.1)$ & 5.4 & 5.4 & weak det. \\
\hline${ }^{34} \mathrm{SO}$ & $\begin{array}{l}3_{4}-2_{3} \\
8_{8}-7_{7}\end{array}$ & $5.6(0.1)$ & 0.39 & $\begin{array}{c}26 \\
197\end{array}$ & $\begin{array}{c}2.42(0.04) \\
<0.009\end{array}$ & 10.22 & 5.52 & no det. \\
\hline \multirow[t]{4}{*}{$\mathrm{SO}_{2}$} & $\begin{array}{c}5_{1,5}-4_{0,4} \\
10_{0,10}-9_{1,9}\end{array}$ & $\begin{array}{c}5.05(0.1) \\
5.1(0.3)\end{array}$ & $\begin{array}{l}0.52 \\
0.33\end{array}$ & $\begin{array}{l}38 \\
43\end{array}$ & $\begin{array}{l}2.33(0.05) \\
1.41(0.06)\end{array}$ & $\begin{array}{l}8.63 \\
8.02\end{array}$ & $\begin{array}{c}3.91 \\
4.0\end{array}$ & \\
\hline & $11_{1,11}-10_{0,10}$ & & & 47 & $<0.006$ & & & no det. \\
\hline & $\begin{array}{l}14_{0,14}-13_{1,13} \\
18_{0,18}-17_{1,17}\end{array}$ & $7.8(0.3)$ & 0.23 & $\begin{array}{l}60 \\
80\end{array}$ & $\begin{array}{c}<0.01 \\
1.89(0.07)\end{array}$ & 7.8 & 7.8 & no det. \\
\hline & $28_{4,24}-28_{3,25}$ & & & 60 & $<0.01$ & & & no det. \\
\hline${ }^{34} \mathrm{SO}_{2}$ & $5_{1,5}-4_{0,4}$ & & & 32 & $<0.006$ & & & no det. \\
\hline \multirow[t]{3}{*}{$\mathrm{OCS}$} & $8-7$ & $6.51(0.02)$ & 0.90 & 11 & $6.20(0.02)$ & 13.10 & 6.55 & $a$ \\
\hline & 13-12 & $4.57(0.03)$ & 1.58 & 39 & 7.33(0.05) & 8.72 & 4.51 & \\
\hline & 19-18 & $5.37(0.02)$ & 1.92 & 35 & $10.46(0.04)$ & 11.03 & 5.01 & \\
\hline $\mathrm{OC}^{34} \mathrm{~S}$ & 8-7 & $7.26(0.03)$ & 0.14 & 7 & $1.13(0.01)$ & 13.63 & 6.92 & \\
\hline \multirow[t]{2}{*}{$\mathrm{H}_{2} \mathrm{~S}$} & $1_{1,0}-1_{0,1}$ & $9.1(0.2)$ & 1.06 & 72 & $12.52(0.6)^{e}$ & 12.50 & 2.76 & $a$ \\
\hline & $2_{2,0}-2_{1,1}$ & $4.15(0.04)$ & 1.26 & 45 & $5.40(0.05)$ & 7.62 & 4.11 & \\
\hline $\mathrm{H}_{2}{ }^{34} \mathrm{~S}$ & $1_{1,0}-1_{0,1}$ & & & 72 & $<0.01$ & & & no det. \\
\hline
\end{tabular}

${ }^{a}$ Self-absorption at $v_{\text {source }},{ }^{b}$ line blend with $\mathrm{H}_{2} \mathrm{CO}$ line from image band.

Table 7. Observed line emissions parameters for IRAS18264-1152.

\begin{tabular}{|c|c|c|c|c|c|c|c|c|}
\hline Species & Transition & $\begin{array}{c}\Delta v \\
\mathrm{~km} \mathrm{~s}^{-1}\end{array}$ & $\begin{array}{c}T_{\mathrm{mb}} \\
\mathrm{K}\end{array}$ & $\begin{array}{c}\sigma \sigma \\
\mathrm{mK}\end{array}$ & $\int_{\mathrm{K} \cdot \mathrm{kmb} \mathrm{s}^{-1}} T_{\mathrm{mb}} \delta \mathrm{V}$ & $\begin{array}{c}\Delta v_{3 \sigma} \\
\mathrm{km} \mathrm{s}^{-1}\end{array}$ & $\begin{array}{c}\Delta v_{1 / 2} \\
\mathrm{~km} \mathrm{~s}^{-1}\end{array}$ & Comment \\
\hline \multirow[t]{3}{*}{$\mathrm{CS}$} & $3-2)$ & $4.19(0.01)$ & 7.2 & 54 & $31.49(0.07)$ & 10.28 & 4.01 & \\
\hline & $5-4$ & $2.97(0.03)$ & 2.26 & 45 & 6.81(0.05) & 7.32 & 2.81 & \\
\hline & $7-6$ & $3.2(0.8) ?$ & $0.6 ?$ & 500 & $1.9(0.5) ?$ & $?$ & $?$ & $b$ \\
\hline \multirow[t]{2}{*}{$\mathrm{C}^{34} \mathrm{~S}$} & $3-2$ & $3.67(0.04)$ & 1.64 & 42 & $6.02(0.05)$ & 8.32 & 3.41 & \\
\hline & $7-6$ & $5.1(0.5)$ & 0.34 & 111 & $1.1(0.1)$ & 3.7 & 4.7 & tentative det. \\
\hline \multirow[t]{4}{*}{ SO } & $3_{4}-2_{3}$ & $3.27(0.02)$ & 3.11 & 44 & $10.38(0.05)$ & 7.32 & 3.21 & \\
\hline & $5_{6}-45$ & $2.98(0.02)$ & 2.33 & 53 & $7.08(0.05)$ & 6.42 & 2.81 & \\
\hline & $65-5_{4}$ & $6.2(0.1)$ & 1.72 & 45 & $11.8(0.2)$ & 11.36 & 7.62 & $a$ \\
\hline & $8_{8}-7_{7}$ & $4.1(0.4)$ & 0.44 & 116 & $1.3(0.1)$ & 2.2 & 4.8 & weak det. \\
\hline \multirow[t]{2}{*}{${ }^{34} \mathrm{SO}$} & $3_{4}-2$ & $3.02(0.2)$ & 0.19 & 39 & $0.60(0.04)$ & 4.01 & 4.01 & \\
\hline & $8_{8}-7_{7}$ & & & 132 & $<0.006$ & & & no det. \\
\hline \multirow[t]{6}{*}{$\mathrm{SO}_{2}$} & $5_{1,5}-4_{0,4}$ & $3.30(0.3)$ & 0.24 & 33 & $0.91(0.05)$ & 5.91 & 5.59 & \\
\hline & $10_{0,10}-9_{1,9}$ & $2.9(0.2)$ & 0.19 & 34 & $0.50(0.03)$ & 3.03 & 3.03 & weak line \\
\hline & $11_{1,11}-10_{0,10}$ & $4.1(0.1)$ & 0.40 & 41 & $1.56(0.04)$ & 7.02 & 3.31 & \\
\hline & $14_{0,14}-13_{1,13}$ & $3.9(0.3)$ & 0.34 & 59 & $0.99(0.07)$ & 3.95 & 3.95 & \\
\hline & $18_{0,18}-17_{1,17}$ & & & 79 & $<0.004$ & & & no det. \\
\hline & $28_{4,24}-28_{3,25}$ & & & 63 & $<0.01$ & & & no det. \\
\hline${ }^{34} \mathrm{SO}_{2}$ & $5_{1,5}-4_{0,4}$ & & & 64 & $<0.01$ & & & no det. \\
\hline \multirow[t]{3}{*}{$\mathrm{OCS}^{2}$} & $\begin{array}{l}8-7,5,4 \\
8-7\end{array}$ & $3.73(0.1)$ & 0.18 & 5 & $0.71(0.01)$ & 9.19 & 3.51 & \\
\hline & $13-12$ & $3.3(0.1)$ & 0.41 & 46 & $1.35(0.05)$ & 4.13 & 3.58 & \\
\hline & $19-18$ & $2.7(0.1)$ & 0.20 & 34 & $0.49(0.03)$ & 3.21 & 3.21 & bad data \\
\hline $\mathrm{OC}^{34} \mathrm{~S}$ & $8-7$ & & & 4 & $<0.001$ & & & no det. \\
\hline \multirow[t]{2}{*}{$\mathrm{H}_{2} \mathrm{~S}$} & $1_{1,0}-1_{0,1}$ & $3.63(0.04)$ & 1.94 & 59 & $7.32(0.06)$ & 7.22 & 3.61 & \\
\hline & $2_{2,0}-2_{1,1}$ & $2.5(0.3)$ & 0.14 & 28 & $0.33(0.03)$ & 5.51 & 2.81 & c \\
\hline $\mathrm{H}_{2}{ }^{34} \mathrm{~S}$ & $1_{1,0}-1_{0,1}$ & $2.7(0.1)$ & 0.35 & 60 & $0.68(0.06)$ & 3.72 & 3.72 & \\
\hline
\end{tabular}

${ }^{a}$ Self-absorption at $v_{\text {source }} ;{ }^{b}$ line blend with $\mathrm{H}_{2} \mathrm{CO}$ line from image band; ${ }^{c}$ unidentified line @ $34.87 \mathrm{~km} \mathrm{~s}^{-1}(216.716 \mathrm{GHz}){ }^{d}+$ other line @ $29.1 \mathrm{~km} \mathrm{~s}^{-1}(135.78223 \mathrm{GHz}), T_{\mathrm{mb}}=0.14 \mathrm{~K}, \delta v=1.1 \mathrm{~km} \mathrm{~s}^{-1}, \int T_{\mathrm{mb}} \delta v=0.17 \mathrm{~K} \cdot \mathrm{km} \mathrm{s}^{-1}$. 
F. Herpin et al.: S-bearing molecules in massive dense cores, Online Material p 6

Table 8. Observed line emissions parameters for IRAS05358+3543.

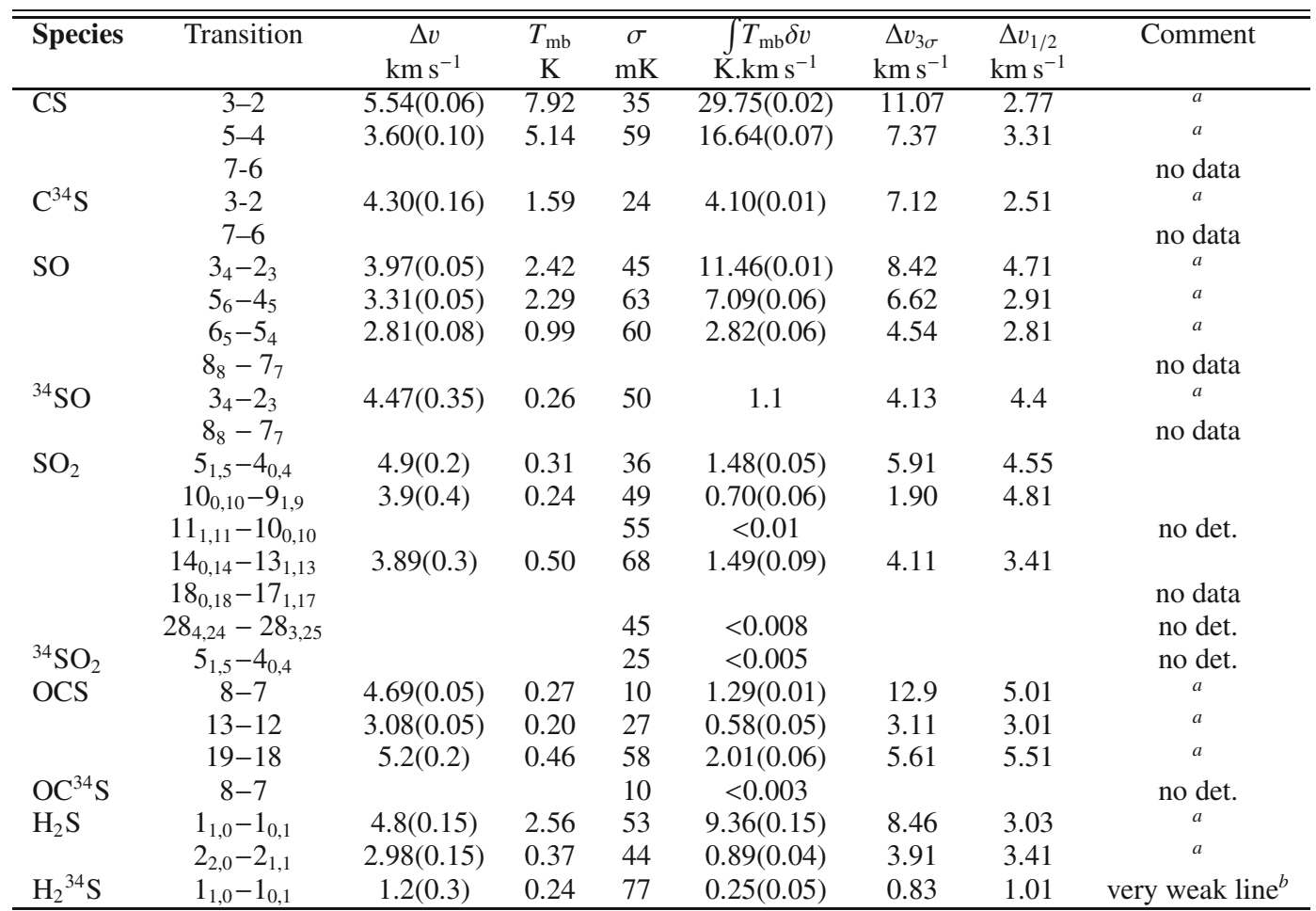

${ }^{a}$ Self-absorption at $v_{\text {source }} ;{ }^{b}$ detected at $2 \sigma$ with two peaks @ -15.57 and $-16.21 \mathrm{~km} \mathrm{~s}^{-1}$.

Table 9. Observed line emissions parameters for IRAS18162-2048.

\begin{tabular}{|c|c|c|c|c|c|c|c|c|}
\hline Species & Transition & $\begin{array}{c}\Delta v \\
\mathrm{~km} \mathrm{~s}^{-1}\end{array}$ & $\begin{array}{c}T_{\mathrm{mb}} \\
\mathrm{K} \\
\end{array}$ & $\begin{array}{c}\sigma \\
\mathrm{mK}\end{array}$ & $\begin{array}{c}\int T_{\mathrm{mb}} \delta v \\
\text { K. } \mathrm{km} \mathrm{s}^{-1}\end{array}$ & $\begin{array}{c}\Delta v_{3 \sigma} \\
\mathrm{km} \mathrm{s}^{-1}\end{array}$ & $\begin{array}{c}\Delta v_{1 / 2} \\
\mathrm{~km} \mathrm{~s}^{-1} \\
\end{array}$ & Comment \\
\hline \multirow[t]{3}{*}{$\mathrm{CS}$} & $3-2$ & & & & & & & no obs. \\
\hline & $5-4$ & $2.93(0.01)$ & 7.41 & 38 & $22.10(0.03)$ & 6.78 & 2.91 & \\
\hline & $7-6$ & $2.46(0.04)$ & 4.05 & 237 & $9.3(0.1)$ & 4.08 & 2.14 & \\
\hline \multirow[t]{2}{*}{$\mathrm{C}^{34} \mathrm{~S}$} & $3-2$ & & & & & & & no obs. \\
\hline & $7-6$ & $2.1(0.3)$ & 0.47 & 116 & $0.87(0.06)$ & 1.17 & 2.63 & \\
\hline \multirow[t]{4}{*}{ SO } & $3_{4}-23$ & $2.24(0.01)$ & 5.27 & 40 & $12.00(0.04)$ & 6.46 & 2.10 & \\
\hline & $5_{6}-4_{5}$ & $2.20(0.01)$ & 5.22 & 45 & $11.31(0.03)$ & 5.42 & 4.84 & \\
\hline & $6_{5}-5_{4}$ & $2.24(0.03)$ & 2.80 & 79 & $6.25(0.08)$ & 4.52 & 1.78 & \\
\hline & $8_{8}-7_{7}$ & $2.2(0.2)$ & 1.04 & 230 & $1.7(0.1)$ & 1.1 & 1.7 & \\
\hline \multirow[t]{2}{*}{${ }^{34} \mathrm{SO}$} & $3_{4}-23$ & & & 38 & $<0.007$ & & & no det. \\
\hline & $8_{8}-7_{7}$ & & & 123 & $<0.006$ & & & no det. \\
\hline \multirow[t]{6}{*}{$\mathrm{SO}_{2}$} & $5_{1,5}-4_{0,4}$ & $3.3(0.1)$ & 0.72 & 34 & $1.86(0.04)$ & 6.46 & 1.68 & \\
\hline & $10_{0,10}-9_{1,9}$ & $1.69(0.07)$ & 0.43 & 33 & $0.75(0.02)$ & 2.39 & 1.75 & $a$ \\
\hline & $\left.11_{1,11}-10_{0,10}\right)$ & $3.86(0.08)$ & 1.14 & 35 & $4.20(0.07)$ & 7.88 & 3.49 & \\
\hline & $14_{0,14}-13_{1,13}$ & $3.63(0.09)$ & 1.02 & 59 & $3.71(0.07)$ & 5.81 & 3.68 & \\
\hline & $18_{0,18}-17_{1,17}$ & $1.2(0.5)$ & 0.13 & 75 & $0.16(0.05)$ & & $3.7 ?$ & tentative det. \\
\hline & $28_{4,24}-28_{3,25}$ & & & 42 & $<0.007$ & & & no det. \\
\hline${ }^{34} \mathrm{SO}_{2}$ & $5_{1,5}-4_{0,4}$ & & & 38 & $<0.007$ & & & no det. \\
\hline \multirow[t]{3}{*}{ OCS } & $8-7$ & $3.5(0.1)$ & 0.12 & 8 & $0.40(0.01)$ & 7.17 & 3.10 & \\
\hline & $13-12$ & $4.7(0.5)$ & 0.23 & 51 & $0.75(0.06)$ & 2.85 & 6.26 & \\
\hline & $19-18$ & $5.12(0.08)$ & 0.55 & 33 & $2.53(0.04)$ & 7.55 & 4.55 & \\
\hline $\mathrm{OC}^{34} \mathrm{~S}$ & 8-7 & & & 9 & $<0.003$ & & & no det. \\
\hline \multirow{2}{*}{$\mathrm{H}_{2} \mathrm{~S}$} & $1_{1,0}-1_{0,1}$ & $3.46(0.02)$ & 2.92 & 50 & $10.00(0.05)$ & 8.0 & 3.36 & $a$ \\
\hline & $2,0-2,1$ & $3.2(0.3)$ & 0.24 & 24 & $0.78(0.07)$ & 4.26 & 2.97 & $b$ \\
\hline $\mathrm{H}_{2}{ }^{34} \mathrm{~S}$ & $1_{1,0}-1_{0,1}$ & $2.3(0.1)$ & 0.60 & 65 & $1.15(0.06)$ & 2.58 & 1.55 & \\
\hline
\end{tabular}

${ }^{a}$ Self-absorption at $v_{\text {source }} ;{ }^{b}$ unidentified lines @ 216.701026 and $216.71 .6 \mathrm{GHz}\left(25\right.$ and $\left.4 \mathrm{~km} \mathrm{~s}^{-1}\right)$. 
F. Herpin et al.: S-bearing molecules in massive dense cores, Online Material $p 7$

Table 10. Opacities derived from modeling the molecular emission of the sources, where "ND" stands for no data.

\begin{tabular}{lcccc}
\hline \hline Lines & W43-MM1 & $\mathbf{1 8 2 6 4}$ & $\mathbf{0 5 3 5 8}$ & $\mathbf{1 8 1 6 2}$ \\
\hline $\mathrm{CS} 3-2$ & 52.0 & 1.6 & 0.45 & $\mathrm{ND}$ \\
$\mathrm{CS} 5-4$ & 65.0 & 0.31 & 0.81 & 0.67 \\
$\mathrm{CS} 7-6$ & 300 & 0.94 & $\mathrm{ND}$ & 2.8 \\
$\mathrm{C}^{34} \mathrm{~S} 3-2$ & 2.8 & 0.18 & 0.09 & $\mathrm{ND}$ \\
$\mathrm{C}^{34} \mathrm{~S} 7-6$ & 16.0 & 0.51 & $\mathrm{ND}$ & 0.41 \\
$\mathrm{OCS} 8-7$ & 6.0 & 0.07 & 0.04 & 0.06 \\
$\mathrm{OCS} 13-12$ & 2.6 & 0.1 & 0.06 & 0.1 \\
$\mathrm{OCS} 19-18$ & 4.9 & 0.12 & 0.07 & 0.2 \\
$\mathrm{OC}^{34} \mathrm{~S} 8-7$ & 0.4 & 0.03 & 0.02 & 0.05 \\
$\mathrm{H}_{2} \mathrm{~S} 1_{1,0}-1_{0,1}$ & 4.2 & 0.53 & 0.21 & 3.9 \\
$\mathrm{H}_{2} \mathrm{~S} 2_{2,0}-2_{1,1}$ & 3.3 & 0.13 & 0.08 & 0.15 \\
$\mathrm{H}_{2}{ }^{34} \mathrm{~S}_{1,0}-1_{0,1}$ & 0.36 & 0.11 & 0.04 & 0.48 \\
$\mathrm{SO} 3_{4}-2_{3}$ & 6.3 & 0.25 & 0.32 & 0.36 \\
$\mathrm{SO} 5_{6}-4_{5}$ & 0.72 & 0.21 & 0.15 & 0.39 \\
$\mathrm{SO}_{5}-5_{4}$ & 0.52 & 0.21 & 0.10 & 0.28 \\
$\mathrm{SO}_{8}-7_{7}$ & 6.8 & 0.33 & $\mathrm{ND}$ & 0.59 \\
${ }^{34} \mathrm{SO}_{4}-2_{3}$ & 0.45 & 0.05 & 0.11 & 0.89 \\
${ }^{34} \mathrm{SO}_{8}-7_{7}$ & 1.6 & 0.26 & $\mathrm{ND}$ & 2.9 \\
$\mathrm{SO}_{2} 5_{1,5}-4_{0,4}$ & 0.42 & 0.06 & 0.03 & 0.08 \\
$\mathrm{SO}_{2} 10_{0,10}-9_{1,9}$ & 0.33 & 0.06 & 0.03 & 0.04 \\
$\mathrm{SO}_{2} 11_{1,11}-10_{0,10}$ & $\mathrm{ND}$ & 0.11 & $\mathrm{ND}$ & 0.13 \\
$\mathrm{SO}_{2} 14_{0,14}-13_{1,13}$ & $\mathrm{ND}$ & 0.21 & 0.23 & 0.42 \\
$\mathrm{SO}_{2} 18_{0,18}-17_{1,17}$ & 6.0 & 0.24 & $\mathrm{ND}$ & 0.22 \\
${ }^{34} \mathrm{SO}_{2} 5_{1,5}-4_{0,4}$ & 0.18 & 0.61 & 0.02 & 0.03 \\
\hline
\end{tabular}

